\title{
Early galaxy growth: mergers or gravitational instability?
}

\author{
A. Zanella ${ }^{1 \star}$, A. Pallottini ${ }^{2}$, A. Ferrara ${ }^{2}$, S. Gallerani ${ }^{2}$, S. Carniani ${ }^{2}$, M. Kohandel $^{2}$, \\ C. Behrens ${ }^{3}$ \\ ${ }^{1}$ Istituto Nazionale di Astrofisica, Vicolo dell'Osservatorio 5, 35122 Padova (Italy) \\ 2 Scuola Normale Superiore, Piazza dei Cavalieri 7, I-56126 Pisa (Italy) \\ 3 Institut für Astrophysik, Georg-August Universität Göttingen, Friedrich-Hundt-Platz 1, 37077, Göttingen (Germany)
}

Accepted XXX. Received YYY; in original form ZZZ

\begin{abstract}
We investigate the spatially-resolved morphology of galaxies in the early Universe. We consider a typical redshift $z=6$ Lyman Break galaxy, "Althæa" from the SERRA hydrodynamical simulations. We create mock rest-frame ultraviolet, optical, and farinfrared observations, and perform a two-dimensional morphological analysis to deblend the galaxy disk from substructures (merging satellites or star-forming regions). We find that the $[\mathrm{CII}] 158 \mu \mathrm{m}$ emitting region has an effective radius $1.5-2.5$ times larger than the optical one, consistent with recent observations. This [CII] halo in our simulated galaxy arises as the joint effect of stellar outflows and carbon photoionization by the galaxy UV field, rather than from the emission of unresolved nearby satellites. At the typical angular resolution of current observations ( $\gtrsim 0.15$ ") only merging satellites can be detected; detection of star-forming regions requires resolutions of $\lesssim 0.05$ ". The [CII]-detected satellite has a $2.5 \mathrm{kpc}$ projected distance from the galaxy disk, whereas the star-forming regions are embedded in the disk itself (distance $\lesssim 1 \mathrm{kpc}$ ). This suggests that multi-component systems reported in the literature, which have separations $\gtrsim 2 \mathrm{kpc}$, are merging satellites, rather than galactic substructures. Finally, the star-forming regions found in our mock maps follow the local $\mathrm{L}_{[\mathrm{CII}]}-\mathrm{SFR}_{\mathrm{UV}}$ relation of galaxy disks, although sampling the low-luminosity, low-SFR tail of the distribution. We show that future JWST observations, bridging UV and [CII] datasets, will be exceptionally suited to characterize galaxy substructures thanks to their exquisite spatial resolution and sensitivity to both low-metallicity and dust-obscured regions that are bright at infrared wavelengths.
\end{abstract}

Key words: galaxies: high-redshift - galaxies: formation - galaxies: evolution galaxies: ISM

\section{INTRODUCTION}

Galaxies in the first billion years of the Universe lifetime undergo a rapid assembly phase and their properties quickly change over time. The period between redshift $z=4-6$ represents a key transition phase from the primordial Universe, when neutral hydrogen was ionized by the first sources $(z>6)$, and the peak of cosmic star formation rate (SFR) density, when galaxies are mature $(z \sim 2-3)$. Studying galaxies at this early epoch is important to understand how they assemble their mass while building up the structures that are commonly observed in local sources (e.g. disk, bulge). In particular, analyzing their morphology provides key insights into their formation and structural evolution.

In the local Universe most of the star-forming galax-

^ E-mail: anita.zanella@inaf.it ies show a central bulge with old stellar populations embedded in a thick disk with spiral arms hosting giant molecular clouds (molecular gas mass $M_{\text {mol }} \sim 10^{5}-10^{7}$ $\mathrm{M}_{\odot}$ ) and star clusters (stellar mass $\mathrm{M}_{\star} \sim 10^{3}-10^{6} \mathrm{M}_{\odot}$, Conselice 2014 and references therein). The bulk of starforming galaxies at $z \sim 1-3$ instead shows irregular morphologies with thin disks dominated by massive star-forming regions $\left(\mathrm{M}_{\star} \sim 10^{7}-10^{9} \mathrm{M}_{\odot}\right.$, size $\left.\lesssim 1 \mathrm{kpc}\right)$ with bright blue colors, typically called "clumps" (Bournaud 2016 and references therein). It is still debated whether clumps are transient features quickly disrupted by their own intense stellar feedback (Genel et al. 2012, Hopkins et al. 2012, Moody et al. 2014, Tamburello et al. 2015, Oklopčić et al. 2017) or if they survive for $\gtrsim 100 \mathrm{Myr}$. In the latter case, they are expected to migrate inward, contribute to the formation of the bulge and the thickening of the disk, playing a key role in galaxy evolution (Immeli et al. 2004, 
Dekel et al. 2009, Ceverino et al. 2012, Inoue et al. 2016, Mandelker et al. 2015, Mandelker et al. 2017). Also the origin of clumps is still under investigation: it is unclear in fact whether they are remnants of merging satellites or if instead they formed in the gas-rich, turbulent disk of galaxies due to gravitational disk instability (Bournaud et al. 2008, Genzel et al. 2008, Genzel et al. 2011, Puech et al. 2009, Wuyts et al. 2014, Guo et al. 2015, Zanella et al. 2015, Ribeiro et al. 2017, Fisher et al. 2017). Different avenues have been followed to tackle this problem, including the analysis of spatially-resolved velocity and Toomre parameter maps (Förster Schreiber et al. 2011, Wisnioski et al. 2011, Genzel et al. 2014, Girard et al. 2018); the characterisation of physical properties (e.g. size, mass, metallicity, stellar populations) of statistical samples of clumps (Guo et al. 2018; Zanella et al. 2019); the investigation of the redshift evolution of the number fraction of clumpy galaxies in the overall star-forming population (Guo et al. 2015; Shibuya et al. 2016). By comparing the fraction of clumpy galaxies at $z \sim 0-3$ with theoretical predictions, Guo et al. (2015) conclude that clumps in galaxies with $\mathrm{M}_{\star} \gtrsim 10^{11} \mathrm{M}_{\odot}$ are likely merger remnants, whereas in lower mass galaxies they form through gravitational instability. Shibuya et al. (2016) extend this analysis to higher redshift, including a sample of Lyman break galaxies (LBGs) at $z \sim 4-8$ observed with the Hubble Space Telescope (HST). They find that the fraction of clumpy galaxies at $z \gtrsim 3$ decreases following the drop of the cosmic SFR density. They conclude that only the theoretical works predicting the in-situ growth of clumps can simultaneously reproduce the fraction of clumpy galaxies observed at low- and high-redshift. Spatially-resolved observations of individual $z \sim 4-6$ galaxies however are needed to determine the physical properties of clumps in the early Universe, confirm their in-situ origin, and understand what is their role in the evolution of the host galaxy. Furthermore, to build a comprehensive picture of galaxy formation, it is important to analyze multiwavelength datasets. Rest-frame ultraviolet (UV) observations tracing unobscured star formation and stellar winds (Heckman et al. 1997, Maraston et al. 2009, Steidel et al. 2010, Faisst et al. 2016) are complementary to far-infrared (FIR) continuum and emission line (e.g. [CII]) data tracing the obscured star formation, gas and dust content (De Looze et al. 2014, Pavesi et al. 2019). In recent years, the exquisite resolution and sensitivity of the Atacama Large Millimeter / submillimeter Array (ALMA) made possible the comparison of the FIR morphology of $z \sim 4-6$ galaxies with the UV one shown by HST. These studies revealed a large fraction of multi-component systems with complex morphology where, in some cases, the UV and FIR emission are even spatially offsetted (Carniani et al. 2018, Le Fèvre et al. 2019, Jones et al. 2020 and references therein). The origin of these substructures is unclear: they could be mergers or galaxies with massive clumps. A detailed multiwavelength study of these systems is important to understand their nature, the fraction of dust-obscured satellites and clumps (that would be missed in UV-based surveys), and in turn understand the contribution of gravitational instability and mergers to galaxy mass assembly and evolution.

As observations progressed, several models of galaxies in the epoch of reionization were developed. In particular cosmological simulations have been used to zoom- in on the structure of high-redshift galaxies and investigate their contribution to the reionization (Katz et al. 2017, Trebitsch et al. 2017, Rosdahl et al. 2018, Hopkins et al. 2018), as well as the role of stellar feedback in the formation and evolution of these primordial sources (Agertz \& Kravtsov 2015, Pallottini et al. 2017a). Simulations have been key also to investigate the chemical enrichment processes in galaxies at high redshift (Maio et al. 2016, Smith et al. 2017, Pallottini et al. 2017b, Lupi et al. 2018, Capelo et al. 2018), and the dust content of such sources (Behrens et al. 2018; Liang et al. 2019).

With this work we aim at bridging simulations and observations to investigate how primeval galaxies form and assemble their mass. Starting from the zoomed-in cosmological simulations of Lyman break galaxies developed by Pallottini et al. 2017b, we create mock rest-frame ultraviolet, optical, and far-infrared observations with the goal of analyzing the structure of these galaxies and relate it to the morphology of $z \sim 6$ observed sources. In particular we compare two stages: in the first the galaxy appears as an undisturbed clumpy disk, in the second it is undergoing a merger. We investigate how the galaxy morphology differs in these two cases and what components are detected when considering different tracers (e.g. rest-frame UV and FIR emission). This paper is organized as follows: in Section 2 we summarize the main characteristics of the zoom-in cosmological simulations adopted in this work; in Section 3 we describe how we created mock rest-frame ultraviolet, optical, and far-infrared observations; in Section 4 we discuss how we analyzed the mock two-dimensional maps and we measured the structural properties of galaxies; in Section 5 we report the results of the analysis; in Section 6 we interpret our results and compare them with those reported in the literature; finally in Section 7 we summarize and conclude. Throughout the paper we use a flat $\Lambda$ CDM cosmology with $\Omega_{\mathrm{m}}=0.3, \Omega_{\Lambda}=0.7$, and $\mathrm{H}_{0}=70 \mathrm{~km} \mathrm{~s}^{-1} \mathrm{Mpc}^{-1}$. We assume a Kroupa (2001) initial mass function (IMF) and, when necessary, we accordingly converted literature results obtained with different IMFs.

\section{GALAXY SIMULATIONS}

The adopted hydrodynamical simulations are fully described in Pallottini et al. (2017b). The simulation is based on a modified version of the adaptive mesh refinement code RAMSES $^{1}$ (Teyssier 2002), in order to evolve a comoving cosmological volume of $(20 \mathrm{Mpc} / h)^{3}$, that is generated with MU$\mathrm{SIC}^{2}$ (Hahn \& Abel 2011). The simulation zooms-in the Lagrangian region of of a dark matter halo of mass $\simeq 3.5 \times$ $10^{11} \mathrm{M}_{\odot}$ at $z \simeq 6$, that hosts the galaxy "Althæa". The gas mass resolution in the zoomed region is $10^{4} \mathrm{M}_{\odot}$ and at $z=6$ it is resolved to spatial scales of $\simeq 30 \mathrm{pc}^{3}$, by adopting a quasi-Lagrangian mass-refinement criterion. In the simulation stars are formed from molecular hydrogen according to a Schmidt-Kennicutt relation (Schmidt 1959; Kennicutt

\footnotetext{
1 https://bitbucket.org/rteyssie/ramses/

2 https://bitbucket.org/ohahn/music/

3 The simulation adopts a fixed resolution in comoving coordinates, thus the physical resolution degrades as the simulation evolve in time, reaching the worst resolution $(30 \mathrm{pc})$ at $z=6$.
} 
Table 1. Physical properties of Althæa in the two stages considered for our analysis: the clumpy disk and the merger with a nearby satellite.

\begin{tabular}{ccccccc}
\hline \hline & $\begin{array}{c}M_{\star} \\
10^{9} \mathrm{M}_{\odot}\end{array}$ & $\begin{array}{c}\mathrm{SFR} \\
\mathrm{M}_{\odot} \mathrm{yr}^{-1}\end{array}$ & $\begin{array}{c}M_{g} \\
10^{9} \mathrm{M}_{\odot}\end{array}$ & $\begin{array}{c}M_{\mathrm{H} 2} \\
10^{7} \mathrm{M}_{\odot}\end{array}$ & $\begin{array}{c}Z \\
Z_{\odot}\end{array}$ & $A_{V}$ \\
\hline Clumpy disk & 7.1 & 49.2 & 1.7 & 2.0 & 0.8 & 1.5 \\
Merging galaxy & 9.9 & 48.1 & 1.8 & 2.4 & 0.7 & 0.9 \\
Satellite & 1.3 & 10.8 & 3.4 & 0.4 & 1.1 & 1.5 \\
\hline
\end{tabular}

Columns: (1) Galaxy. (2) Stellar mass. (3) Star formation rate. (4) Total gas mass. (5) Molecular gas mass. (6) Metallicity. (7) Extinction.

1998). The abundance of the molecular hydrogen is computed using time dependent chemical network implemented using the $\mathrm{KROME}^{4}$ package (Grassi et al. 2014; Bovino et al. 2016). Stellar feedback includes supernova explosions, radiation pressure, and winds from massive stars (Pallottini et al. 2017b); the model also accounts for the blastwave propagation inside molecular clouds, and the thermal and turbulent energy content of the gas is modelled similarly to Agertz \& Kravtsov (2015). Stellar energy inputs and chemical yields are calculated via STARBURST99 (Leitherer et al. 1999) assuming a Kroupa (2001) IMF for the star clusters. In this simulation a spatially uniform interstellar radiation field is considered, and its intensity scales with the star formation rate of the galaxy (see for details Pallottini et al. 2019).

Althæa appears as a typical $z \geq 6$ LBG (Behrens et al. 2018, 2019), following the SFR $-M_{\star}$ relation observed at high- $z$ (Jiang et al. 2016). At the earliest epochs, it is constituted by a small disk surrounded by several substructures (size $<100 \mathrm{pc}$ ) - typically coinciding with molecular cloud complexes (Leung et al. 2019) - and it is fed with gas through filaments (Kohandel et al. 2019). As time passes, the disk grows in size and mass thanks to in-situ starformation and mergers with satellites which are disrupted and embedded in the disk (Gelli et al., subm. 2020).

For this work we focus on two specific evolutionary stages with a morphological distinct structure (see also Kohandel et al. 2019; Kohandel et al., subm. 2020): (a) a clumpy disk, found at $z=7.2$, with a total stellar (molecular gas) mass $M_{\star}=7.1 \times 10^{9} M_{\odot}\left(M_{\mathrm{H} 2}=2.0 \times 10^{7} M_{\odot}\right)$, a star formation rate $^{5} \mathrm{SFR}=49.2 M_{\odot} \mathrm{yr}^{-1}$, and metallicity $Z=0.8 Z_{\odot}$; (b) a merger, found at $z=6.47$, with a total stellar (molecular gas) mass $M_{\star}=9.9 \times 10^{9} M_{\odot}\left(M_{\mathrm{H} 2}=2.4 \times 10^{7} M_{\odot}\right)$, a star formation rate $\mathrm{SFR}=48.1 M_{\odot} \mathrm{yr}^{-1}$, and metallicity $Z=0.7 Z_{\odot}$ (Table 1$)$. For a fair observational comparison, we redshifted both of them to $z=6$. In the rest of the paper we will consider these two stages as independent sources.

\section{MULTI-WAVELENGTH MOCK MAPS}

To compare the morphology and structural parameters of our simulated galaxies with actual $z \sim 5-7$ observations, the first step is to create synthetic continuum and emission line maps. In particular we aim at reproducing typical

\footnotetext{
4 https://bitbucket.org/tgrassi/krome

5 In the present work, the star formation rate is computed accounting for stars with age $t_{\star}<30 \mathrm{Myr}$.
}

$H S T$ optical images (bands $z^{\prime}, Y, J$, and $H$ ), ALMA submillimeter continuum (Band 6) and emission line ([CII]) two-dimensional (2D) maps, as well as realistic near- and mid-infrared JWST observations.

First, starting from the simulated galaxy, we generated mock continuum and emission line images by using SKIRT (Camps \& Baes 2015) and CLOUDY (Ferland et al. 2017) respectively (Section 3.1). Then we added observational artifacts to mimic typical UV and FIR high-redshift observations (Sections 3.2).

\subsection{Continuum and emission lines modelling}

Continuum emission is generated by using SKIRT $^{6}$ (Baes \& Camps 2015; Camps \& Baes 2015), a Monte Carlo based code that computes the radiative transfer process in dusty media. The setup adopted here is similar to Behrens et al. (2018), and we summarize it as follows. The spatial distribution of the light sources is taken from the position of the stellar clusters in Althæa; for each cluster, we use its metallicity and age to compute the stellar spectral energy distribution (SED), by adopting the Bruzual \& Charlot (2003) models, and the same Kroupa (2001) IMF used in the simulation.

In Pallottini et al. (2017b), the metal content of the gas is evolved accounting for supernovae and processed ejecta from stellar winds, starting from a metallicity $Z=10^{-3} \mathrm{Z}_{\odot}$ floor, as expected from a preenrichment scenario (Tornatore et al. 2007; Pallottini et al. 2014; Maiolino \& Mannucci 2019). Dust is not directly traced and we adopt dust-to-metal ratio $f_{\mathrm{d}}=0.08$. such value is found in Behrens et al. (2018) in order to have an observed UV and FIR SED comparable to high-redshift observations (Laporte et al. 2017). Note that in the Milky Way $f_{\mathrm{d}}=0.3$ and typically $f_{\mathrm{d}} \simeq 0.2$ in local galaxies (De Looze et al. 2020), while at high-redshift the value is much more uncertain (Wiseman et al. 2017). Dust composition and grain size distribution is set to mimic the MilkyWay (Weingartner \& Draine 2001), and we assume a dust emissivity $\beta_{d}=2$.

We use CLOUDY ${ }^{7}$ (Ferland et al. 2017) to compute the line emission for [CII] and CO roto-vibrational transitions. Similarly to (Pallottini et al. 2019), we use grids of CLOUDY models for density, metallicity, radiation field intensity, as a function of the column density. We account for the turbulent and clumpy structure of the interstellar medium (ISM), by parameterizing the underlying distribution as a function of the gas Mach number (Vallini et al. 2017, 2018). With respect to Pallottini et al. (2019), here the radiation field is assumed to be uniform and non-ionizing, thus photoevaporation effects are not fully included: such effect can both modify the emission lines strength (Vallini et al. 2017) and the $\mathrm{H}_{2}$ - and thus star - formation (Decataldo et al. 2019). While in general line emission is sensitive to adopted models (Olsen et al. 2018), the resulting [CII] flux is more robust to changes of assumptions (Lupi et al. 2020). Note that the considered FIR lines are optically thin given the column densities found in the ISM of Althæa, thus no further dust

\footnotetext{
6 version 8.0, http://www. skirt.ugent.be

7 C17.01 https://www.nublado.org/
} 
Table 2. Parameters of the mock observations

\begin{tabular}{|c|c|c|c|c|}
\hline $\begin{array}{c}\text { Telescope } \\
(1)\end{array}$ & $\begin{array}{l}\text { Band } \\
\text { (2) }\end{array}$ & $\begin{array}{c}\lambda_{\mathrm{c}} \\
(\mu \mathrm{m}) \\
(3)\end{array}$ & $\begin{array}{c}\text { Angular resolution } \\
\left(\operatorname{arcsec}^{2}\right) \\
(4)\end{array}$ & $\begin{array}{r}\text { Depth } \\
(5)\end{array}$ \\
\hline$H S T$ & ACS/F850LP $\left(z^{\prime}\right)$ & 0.9 & $\begin{array}{l}0.13 \times 0.13 \\
0.04 \times 0.04\end{array}$ & $\begin{array}{l}29.0 \\
29.0\end{array}$ \\
\hline$H S T$ & WFC3/F105W $(Y)$ & 1.1 & $\begin{array}{l}0.13 \times 0.13 \\
0.04 \times 0.04\end{array}$ & $\begin{array}{l}29.0 \\
29.0\end{array}$ \\
\hline$H S T$ & WFC3/F125W $(J)$ & 1.2 & $\begin{array}{l}0.13 \times 0.13 \\
0.04 \times 0.04\end{array}$ & $\begin{array}{l}29.0 \\
29.0\end{array}$ \\
\hline$H S T$ & WFC3/F160W $(H)$ & 1.5 & $\begin{array}{l}0.15 \times 0.15 \\
0.05 \times 0.05\end{array}$ & $\begin{array}{l}29.0 \\
29.0\end{array}$ \\
\hline$J W S T$ & NIRCam/F444W (near-IR) & 4.4 & $\begin{array}{l}0.15 \times 0.15 \\
0.05 \times 0.05\end{array}$ & $\begin{array}{l}29.0 \\
29.0\end{array}$ \\
\hline$J W S T$ & MIRI/F770W (mid-IR) & 7.7 & $\begin{array}{l}0.24 \times 0.24 \\
0.08 \times 0.08\end{array}$ & $\begin{array}{l}29.0 \\
29.0\end{array}$ \\
\hline ALMA & Band 6 (continuum) & 1100.0 & $\begin{array}{l}0.18 \times 0.12 \\
0.05 \times 0.04\end{array}$ & $\begin{array}{l}3.4 \\
4.7\end{array}$ \\
\hline ALMA & Band 6 ([CII $])$ & 1100.0 & $\begin{array}{l}0.18 \times 0.12 \\
0.05 \times 0.04\end{array}$ & $\begin{array}{l}19.0 \\
18.0\end{array}$ \\
\hline
\end{tabular}

Columns: (1) Telescope. (2) Camera and filter. (3) Central wavelength of the filter. (4) Angular resolution achieved in the mock observations for the low- (top) and high-resolution (bottom) case. See Section 3 for details. (5) Depth of the observations. We report the limiting magnitude (in AB mag) defined as $5 \sigma$ sky noise in a 0.25 "radius aperture for the $H S T$ and $J W S T$ observations. We report the

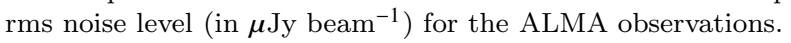

attentuation is needed. For a fair comparison, we set the spatial resolution of the continuum and lines at $25 \mathrm{pc}$ for both the clumpy disk and merger case, and we redshifted both of them to $z=6$. In both snapshots the galactic disk is seen face-on.

\subsection{Mimicking observational artefacts}

To properly reproduce actual observations we also need to mimic the image broadening due to the limited spatial resolution (i.e. diffraction limit), the pixelization of the detectors, and the presence of noise that limits the depth of the data.

We reproduced the case of spatially-resolved observations of galaxies where a typical image quality of $0.5-1 \mathrm{kpc}$, corresponding to 0.1 " -0.2 " at $z \sim 6$, is reached (depending on the observing band, e.g. Grogin et al. 2011, Shibuya et al. 2015). We also considered the ideal case of a galaxy observed with a resolution of $\sim 0.25 \mathrm{kpc}$, corresponding to $\sim 0.05$ " at this redshift. Currently this resolution is beyond the diffraction limit of $H S T$ and is only achievable in moderately lensed sources (e.g. with magnification $\mu \gtrsim 10$, Knudsen et al. 2016, Bradač et al. 2017), or in the submillimeter with ALMA. However in the near future, the bluest JWST/NIRCam filters at wavelength $\lambda \lesssim 1.5 \mu \mathrm{m}$ (corresponding to the $H S T$ ones considered in this paper) will allow the community to achieve such a high spatial resolution also in the optical. Throughout the paper we will refer to the maps with $\sim 0.15$ " resolution as the "low-resolution" case and to the ones with $\sim 0.05$ " resolution as the "high-resolution" case.

We adopted the two following procedures to create mock maps in the optical and infrared (HST, JWST), and at submillimeter wavelengths (ALMA). To obtain the final HST and JWST images we smoothed the original-resolution maps with a Gaussian kernel (Table 2). To account for the pixelization of the detector we resampled the smoothed maps to a pixel scale of 0.03 " pixel $^{-1}$ that can be achieved in $H S T$ imaging when dithering (e.g. Zanella et al. 2019). Adopting a larger pixel scale as in some studies (e.g. 0.06" pixel ${ }^{-1}$, Brammer et al. 2012), does not affect our results. Finally, we have added random noise to reproduce the sensitivity of data taken in the commonly observed cosmological fields (e.g. CANDELS survey, Grogin et al. 2011). The $5 \sigma$ limiting magnitude of our mock optical and infrared images is $\sim 29 \mathrm{AB}$ mag considering a point-source and an aperture with $\sim 0.25$ " radius. The original-resolution images as well as the mock maps obtained for our clumpy disk and merger are shown in Figures 1 and 2.

To reproduce the limited angular resolution, pixelization and noise of sub-millimeter images instead we used $\mathrm{CASA}^{8}$, the observing simulator of ALMA (McMullin et al. 2007). We gave as input the original-resolution continuum and emission line models and generated $u v$ data with the simobserve task. Since in this work we do not focus on the kinematical properties of galaxies, but rather on their morphology, we did not produce hyperspectral cubes (including spatial and velocity information). We directly fed CASA with the $2 \mathrm{D}[\mathrm{CII}]$ models integrated over a line width of $100 \mathrm{~km}$ $\mathrm{s}^{-1}$ (cfr with Kohandel et al., subm. 2020). We then imaged the simulated observations with the simanalyze task. We adopted different configurations, in order to achieve $\sim 0.15$ " and $\sim 0.05 "$ angular resolutions (Table 2). We set the observing time to 10 hours, as this is a typical integration time for high-redshift observations (e.g. Jones et al. 2017, Laporte et al. 2017, Carniani et al. 2018). Imaging was performed using a Briggs weighting scheme (ROBUST $=-0.5$ ) which gives a good trade-off between resolution and sensitivity.

\section{MORPHOLOGICAL STUDY}

The original-resolution maps of our clumpy galaxy and merger do not simply appear as smooth disks, but they rather show several substructures (Figures 1,2). In this work we aim at understanding whether these structures are still detected in realistic mock images that include the effects of limited spatial resolution and sensitivity.

We also investigate how the structural parameters of the galaxy disk (e.g. effective radius, Sérsic index) depend on the resolution and observing band used to measure them. In this Section we discuss the method that we used to deblend the substructures from the underlying disks, how we measured their continuum and emission line fluxes (with associated uncertainties) as well as the properties of the disks. We call "substructures" all the significant detections that depart from a smooth stellar disk (e.g. merging satellites, star-forming clumps), similarly to Guo et al. (2018) and Zanella et al. (2019).

\subsection{Flux measurements}

The procedure that we use to detect the substructure and disentangle it from the underlying galaxy disk is analogous

\footnotetext{
8 v5 https://casa.nrao.edu/
} 


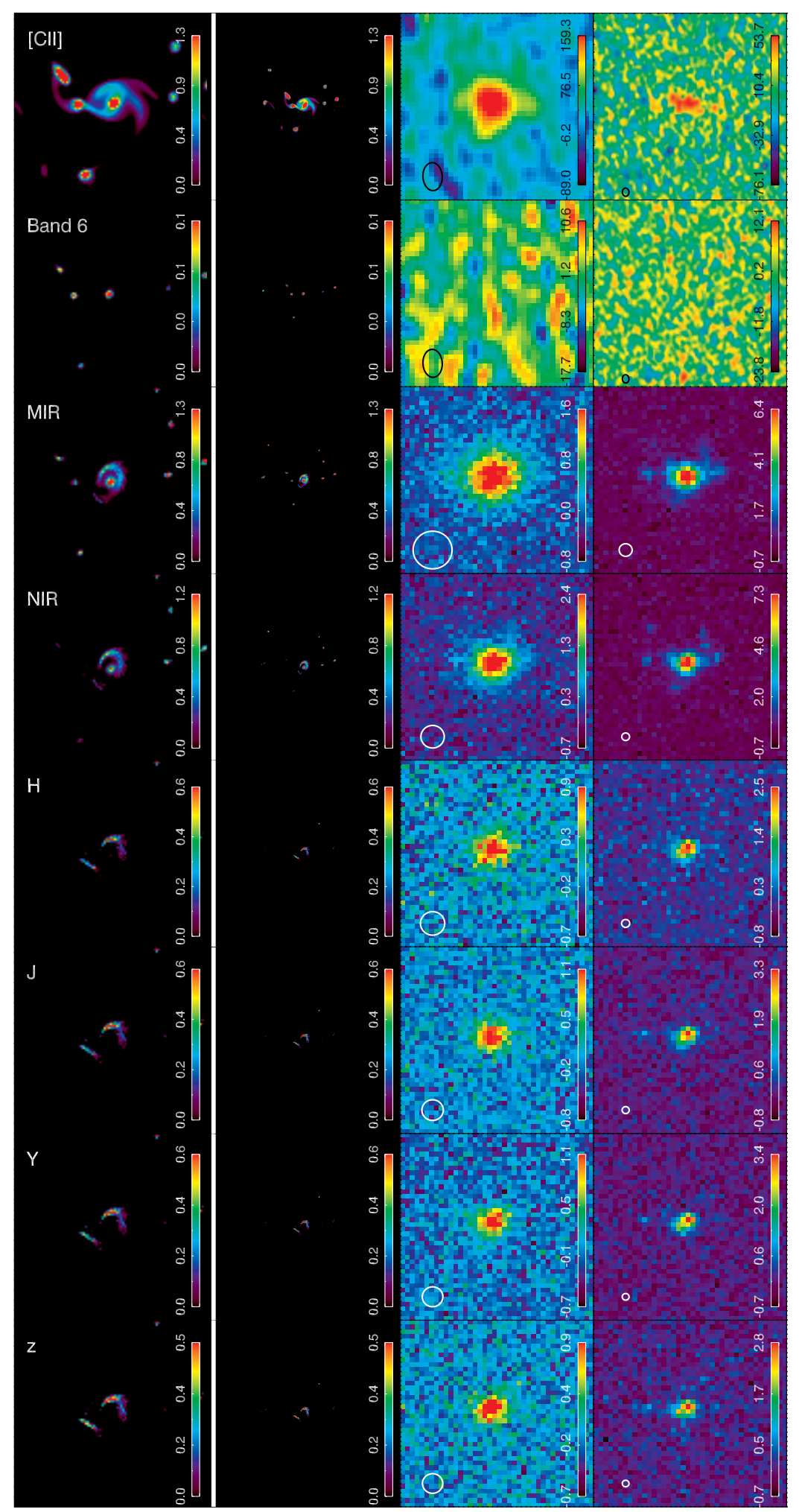

Figure 1. Maps of Althæa in the clumpy disk stage. From top to bottom: different observing bands are shown. HST/ACS F850LP $\left(z^{\prime}\right), H S T$ /WFC3 F105W ( $Y$, F125W $(J)$, F160W $(H)$; JWST/NIRCam F444W (NIR), JWST/MIRI F770W (MIR); ALMA Band 6 continuum and the $[\mathrm{CII}]$ pseudo-narrow band emission line map. From left to right: maps with different spatial resolutions are shown, in particular the nominal resolution from the simulation (first two panels), the typical resolution of current observations ( 0.15" - 0.2", third panel), and the higher resolution currently achievable with ALMA and/or in lensed systems ( 0.05", considering a magnification factor $\mu \sim 10$, fourth panel). The stamps in the leftmost column have a size of $0.2 " \times 0.2 "(\sim 1.1 \times 1.1 \mathrm{kpc}$ at $z \sim 6)$, whereas the other stamps have a size of 0.6 " $\times 0.6 "(\sim 3.4 \times 3.4 \mathrm{kpc}$ at $z \sim 6)$. The color bars report the flux values in units of $10^{-3} \mu \mathrm{Jy}$ for the $H S T$ and $J W S T$ maps, and $\mu \mathrm{Jy}_{\text {beam }}{ }^{-1}$ for the ALMA maps. The white and black circles in the bottom left corner of the maps indicate the spatial resolution of the observations (the full width at half maximum of the point spread function for the HST and JWST maps, the beam for the ALMA bands). 


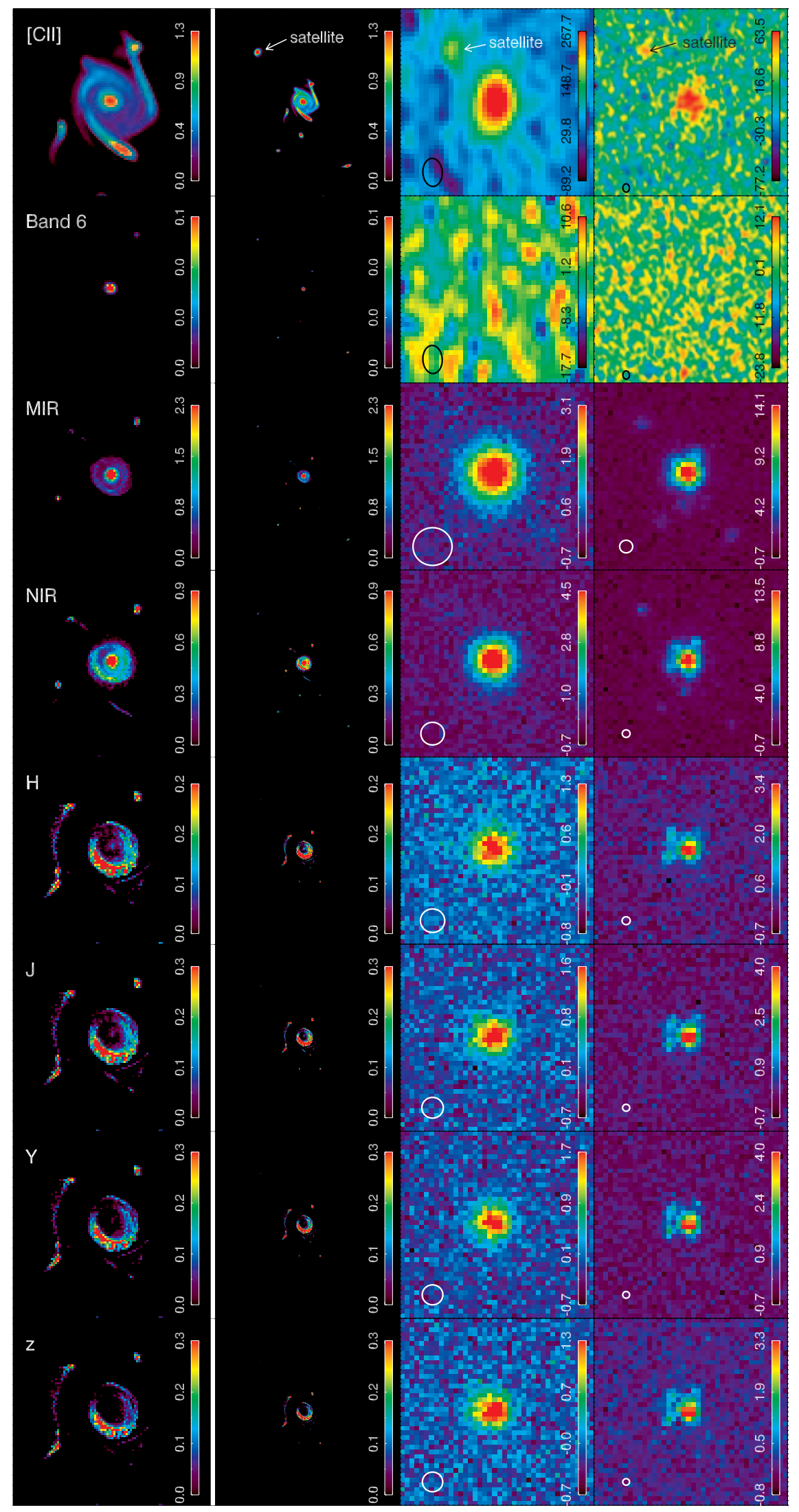

Figure 2. Maps of Althæa in the merger stage. Colors and symbols are as in Figure 1. 
Table 3. Structural parameters.

\begin{tabular}{|c|c|c|c|c|c|c|c|c|c|}
\hline $\begin{array}{c}\text { Galaxy } \\
\text { (1) }\end{array}$ & $\begin{array}{l}\text { Resolution } \\
\text { (2) }\end{array}$ & $\begin{array}{c}\text { Component } \\
\text { (3) }\end{array}$ & $\begin{array}{c}\mathrm{F}_{z} \\
(\mu \mathrm{Jy}) \\
(4)\end{array}$ & $\begin{array}{c}\mathrm{F}_{Y} \\
(\mu \mathrm{Jy}) \\
(5)\end{array}$ & $\begin{array}{c}\mathrm{F}_{J} \\
(\mu \mathrm{Jy}) \\
(6)\end{array}$ & $\begin{array}{c}\mathrm{F}_{H} \\
(\mu \mathrm{Jy}) \\
(7)\end{array}$ & $\begin{array}{c}\mathrm{F}_{\text {near-IR }} \\
(\mu \mathrm{Jy}) \\
(8)\end{array}$ & $\begin{array}{c}\begin{array}{c}F_{\text {mid-IR }} \\
(\mu \mathrm{Jy}) \\
(9)\end{array} \\
\end{array}$ & $\begin{array}{c}\mathrm{F}_{[\mathrm{CII}]} \\
(\mu \mathrm{Jy}) \\
(10)\end{array}$ \\
\hline \multirow[t]{4}{*}{ Clumpy } & Low-resolution & Disk & $0.051 \pm 0.010$ & $0.057 \pm 0.010$ & $0.063 \pm 0.010$ & $0.059 \pm 0.010$ & $0.210 \pm 0.010$ & $0.261 \pm 0.010$ & $614.3 \pm 131.1$ \\
\hline & High-resolution & Disk & $0.052 \pm 0.006$ & $0.054 \pm 0.006$ & $0.057 \pm 0.005$ & $0.055 \pm 0.005$ & $0.189 \pm 0.011$ & $0.244 \pm 0.003$ & $<806.0$ \\
\hline & & Substructure 1 & $<0.003$ & $<0.003$ & $<0.003$ & $<0.003$ & $0.005 \pm 0.001$ & $<0.009$ & $<29.0$ \\
\hline & & Substructure 2 & $<0.035$ & $<0.041$ & $<0.041$ & $<0.039$ & $<0.026$ & $0.189 \pm 0.011$ & $<251.2$ \\
\hline \multirow[t]{7}{*}{ Merger } & Low-resolution & Disk & $0.095 \pm 0.021$ & $0.115 \pm 0.018$ & $0.129 \pm 0.019$ & $0.109 \pm 0.022$ & $0.384 \pm 0.030$ & $0.506 \pm 0.095$ & $787.8 \pm 144.8$ \\
\hline & & Substructure & $<0.005$ & $<0.003$ & $<0.005$ & $<0.004$ & $0.009 \pm 0.003$ & $<0.027$ & $106.7 \pm 17.8$ \\
\hline & High-resolution & Disk & $0.078 \pm 0.006$ & $0.091 \pm 0.006$ & $0.102 \pm 0.006$ & $0.095 \pm 0.005$ & $0.360 \pm 0.008$ & $0.466 \pm 0.003$ & $567.0 \pm 135.0$ \\
\hline & & Substructure 1 & $0.005 \pm 0.001$ & $0.006 \pm 0.001$ & $0.007 \pm 0.001$ & $0.007 \pm 0.001$ & $0.007 \pm 0.001$ & $0.010 \pm 0.003$ & $<28.6$ \\
\hline & & Substructure 2 & $0.006 \pm 0.001$ & $0.007 \pm 0.001$ & $0.007 \pm 0.001$ & $0.007 \pm 0.001$ & $0.007 \pm 0.001$ & $0.010 \pm 0.003$ & $<28.8$ \\
\hline & & Substructure 3 & $<0.004$ & $<0.004$ & $<0.004$ & $<0.003$ & $0.008 \pm 0.001$ & $0.012 \pm 0.003$ & $32.0 \pm 9.6$ \\
\hline & & Substructure 4 & $<0.004$ & $<0.004$ & $<0.004$ & $<0.003$ & $0.008 \pm 0.001$ & $0.013 \pm 0.002$ & $49.4 \pm 13.9$ \\
\hline Galaxy & Resolution & Component & $\begin{array}{l}\mathrm{R}_{\mathrm{e}, z} \\
(\mathrm{pc})\end{array}$ & $\begin{array}{l}\mathrm{R}_{\mathrm{e}, Y} \\
(\mathrm{pc})\end{array}$ & $\begin{array}{l}\mathrm{R}_{\mathrm{e}, J} \\
(\mathrm{pc})\end{array}$ & $\begin{array}{l}\mathrm{R}_{\mathrm{e}, H} \\
(\mathrm{pc})\end{array}$ & $\begin{array}{l}\mathrm{R}_{\mathrm{e}, \text { near-IR }} \\
\quad(\mathrm{pc})\end{array}$ & $\begin{array}{c}\mathrm{R}_{\mathrm{e}, \mathrm{mid}-\mathrm{IR}} \\
(\mathrm{pc})\end{array}$ & $\begin{array}{c}\mathrm{R}_{\mathrm{e},[\mathrm{CII}]} \\
(\mathrm{pc})\end{array}$ \\
\hline \multirow[t]{2}{*}{ Clumpy disk } & Low-resolution & Disk & $246 \pm 37$ & $264 \pm 40$ & $278 \pm 42$ & $274 \pm 41$ & $442 \pm 88$ & $456 \pm 91$ & $733 \pm 147$ \\
\hline & High-resolution & Disk & $303 \pm 45$ & $300 \pm 45$ & $295 \pm 44$ & $293 \pm 44$ & $350 \pm 52$ & $346 \pm 52$ & $751 \pm 150$ \\
\hline \multirow[t]{2}{*}{ Merger } & Low-resolution & Disk & $398 \pm 60$ & $416 \pm 62$ & $423 \pm 63$ & $363 \pm 54$ & $399 \pm 80$ & $398 \pm 80$ & $629 \pm 126$ \\
\hline & High-resolution & Disk & $295 \pm 44$ & $300 \pm 45$ & $308 \pm 46$ & $326 \pm 49$ & $348 \pm 70$ & $284 \pm 57$ & $536 \pm 107$ \\
\hline
\end{tabular}

Columns: (1) Galaxy: clumpy disk or merger. (2) Resolution: low- ( 0.15") or high-resolution ( 0.05"). (3) Component: galaxy disk or substructure. (4) - (10) Flux or effective radius of the disk measured in the given band.

to that described in Zanella et al. (2019). In brief, we modelled with GALFIT (Peng et al. 2010) the 2D light profile of the optical, infrared, and sub-millimeter maps independently. We adopted a single Sérsic profile and we subtracted the best-fit model from each map. With this first step we could understand whether the galaxy disk could be considered smooth or if additional structures would appear in the residuals. By using SEXTRACTOR (Bertin \& Arnouts 1996) independently on each residual map, we identified additional substructures and matched their coordinates. We considered that two detections were matched if their offset in different maps were smaller than the FWHM of the PSF $(\sim 0.15$ " in the low-resolution case, 0.05 " in the high-resolution case). We created a final catalog with the coordinates of all the identified substructures.

We estimated the flux of the substructures and diffuse disk in the different continuum and [CII] maps as follows. We fitted again the 2D light profile of our galaxies considering simultaneously a Sérsic profile to model the disk component and additional PSF or Sérsic profiles at the location of the substructures detected with SEXTRACTOR. We considered Sérsic instead of PSF profiles only if the substructure is resolved (i.e. its effective radius is larger than the FWHM of the PSF in the considered band). We found only one resolved substructure (Section 5.2). Following Zanella et al. (2019), we used the fitting algorithm GALFITM (Vika et al. 2013) that allows to simultaneously fit multiple images of the same galaxy taken at different wavelengths. After the subtraction of the best-fit model, we visually inspected all the residuals to verify the reliability of the fits. We show the results of our fitting procedure in Appendix A.

At the redshift of our targets $(z=6)$, the $H S T$ /WFC3 $z^{\prime}$ bandpass includes the Ly $\alpha$ emission, while the $J W S T$ /NIRCam F444W bandpass includes the $\mathrm{H} \alpha$ line. The UV data produced by SKIRT do not include line emission (see Section 3.1), thus no line subtraction is needed to obtain clean continuum maps. Similarly, this reasoning applies to the ALMA continuum and [CII] emission, and therefore the fluxes estimated with the described procedure do not need further corrections.

\subsection{Size measurements}

The galaxy disks are fitted with a Sérsic profile and therefore we can also measure their effective radius. In the case of isolated galaxies the disks are barely resolved in the optical $H S T$ bands and unresolved in the infrared JWST images that have coarser resolution. They are always resolved in the [CII] maps (Section 5.1). When fitting the images, GALFITM convolves the Sérsic model by an input PSF and returns the deconvolved effective radius. For this reason some of the effective radii reported in Table 3 are smaller than the angular resolution of the mock observations. Since for the same galaxy GALFITM estimates comparable disk radii irrespective of the angular resolution of the observation - the deconvolution procedure can be seen as reliable. To further check whether the limited sensitivity of our mock observations strongly affects the size estimates, we ran GALFITM on noiseless maps. Given the depth of our mock observations, we do not find systematic differences and in all bands the measured effective radii are consistent within the uncertainties with those estimated from the noisy maps.

When performing the fits we let GALFITM free to vary the Sérsic index of the disks. We find that the clumpy disk has Sérsic index $n \sim 1.5$ in all bands. The merger instead has a Sérsic index $n \sim 1$ in the optical bands, $n \sim 2$ in the infrared bands, and $n \sim 0.5$ in the [CII] map. These results do not depend on the angular resolution of our images. Often in the literature, to limit the number of free parameters especially in the case of low signal-to-noise ratio $(\mathrm{S} / \mathrm{N})$, an exponential disk $(n=1)$ is fit. We checked that if we fit the disks adopting exponential profiles, the effective radii remain consistent with the case of a free Sérsic model, within the uncertainties (we find changes $\lesssim 30 \%$ and no systematic trends with the observing band, Figure B1). Finally, we measured the [CII] effective radius by fitting the line visibilities with the CASA task UVMODELFIT, adopting an exponential profile, following the procedure described by Fujimoto et al. (2020). We obtained radii that are fully consistent with the ones measured with GALFIT in the image plane. Throughout the rest of the paper we report the GALFIT measurements, so to 

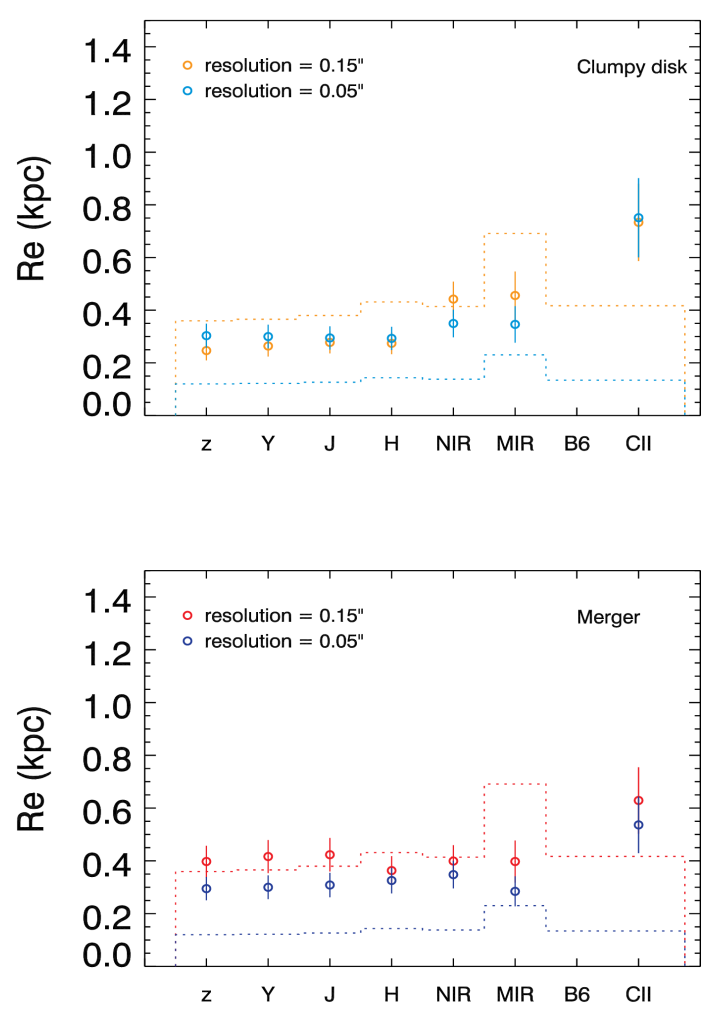

Figure 3. Effective radius of the galaxy disk in different observing bands and with different spatial resolution. Top: Clumpy galaxy case. Bottom: merger case. The radius measured in the mock observations with $\sim 0.15$ " (yellow and red open circles for the clumpy galaxy and merger case respectively), and $\sim 0.05$ " (cyan and blue open circles for the clumpy galaxy and merger case respectively) resolution are reported. The dashed lines show the effective resolution (PSF's FWHM/2) of the mock observations in each band.

adopt consistent methods for optical, infrared, and submillimeter datasets.

\subsection{Estimate of the flux and size uncertainties}

To estimate the uncertainties associated to our flux and size measurements we performed 1000 Monte Carlo simulations. We injected a fake PSF or Sérsic profile at the time on top of each map. The structural parameters of these components were randomly chosen in the range spanned by the substructures and disks in our mock observations. We then treated these images with the same procedure detailed in Section 4.1. To determine the uncertainties associated to the flux of the substructures, we divided the simulated PSFs (or Sérsic profiles) in bins based on the contrast between their luminosity and that of the underlying disk, at the location of the substructure. This was a necessary step because the accuracy of GALFITM in estimating the flux largely depends on the contrast with respect to the underlying disk. For each injected PSF we computed the difference between the known input flux and the one retrieved by GALFITM, in each contrast bin. The standard deviation of the sigma clipped distribution of these differences gave us the flux uncertainties.

Given the fluxes estimated by GALFITM and the associated uncertainties, we determined the $\mathrm{S} / \mathrm{N}$ and considered as detections only the substructures with $\mathrm{S} / \mathrm{N} \gtrsim 3$. If in a given band we obtained a $\mathrm{S} / \mathrm{N}<3$, we calculated a $3 \sigma$ upper limit based on the estimated uncertainty. Using $3 \sigma$ upper limits when studying $H S T$ data is standard in the literature. For ALMA data some works use $3 \sigma$ upper limits, whereas others prefer more conservative $5 \sigma$ limits due to the correlated noise of interferometric observations. In the rest of this work we consider $3 \sigma$ limits also for the sub-millimeter bands, as most of the substructures detected in ALMA are also detected in at least one optical band. All but one structure would still be detected if we were to consider $5 \sigma$ limits instead and our results would not change.

The uncertainties on the effective radii of the Sérsic components were also derived by considering Monte Carlo simulations, with the same procedure adopted for flux uncertainties.

\section{RESULTS}

In this Section we discuss the structural properties (i.e. effective radius, Sérsic index) of the clumpy disk and merger, and compare them with observational results. Furthermore, we investigate the presence and detectability of disks substructures, depending on spatial resolution and spectral band.

\subsection{Spatially-integrated galaxy properties}

Our galaxies are clearly detected $(\mathrm{S} / \mathrm{N} \gtrsim 5)$ in the optical and infrared bands, as well as in the [CII] emission line maps, irrespective of the resolution of the observations. Instead, they are undetectable in the sub-millimeter continuum maps (e.g. Band 6). This is consistent with literature results, where the continuum at $\sim 158 \mu \mathrm{m}$ is often undetected, even when the [CII] is observed with high S/N (Capak et al. 2015, Tamura et al. 2019, Bakx et al. 2020).

We fitted the 2D light profile of the clumpy disk and merger using a Sérsic profile (Section 4.2) and determined the effective radius of the disks. We find that they both have $R_{\mathrm{e}} \sim 300 \mathrm{pc}$ in the optical and infrared bands (Figure 3 ). The galaxies are marginally resolved or unresolved (especially in the JWST/MIRI band) in the low-resolution case, whereas they are resolved at high-resolution. The disk size estimate does not vary, within the uncertainties, when changing the angular resolution of the observations, indicating that the fits are robust when the sources are detected with $\mathrm{S} / \mathrm{N} \gtrsim 5$ at angular resolution $<0.15$ ". The effective radius measured from the $[\mathrm{CII}]$ maps instead is systematically $\sim 1.5-2.5$ times larger than the optical one and ranges between $600 \mathrm{pc}$ (for the merger) and $700 \mathrm{pc}$ (for the clumpy disk).

The fact that [CII] sizes are systematically larger than the optical ones has been already reported (Carniani et al. 2017, Fujimoto et al. 2019, Ginolfi et al. 2020). Recently the ALPINE survey observed a sample of $z=4-6$ galaxies and found, on a statistical basis, that the [CII] sizes are $\sim 2-3$ times larger than the rest-frame UV sizes measured in the $z$ band, and $\sim 1.5-2$ times larger than the rest-frame opti- 

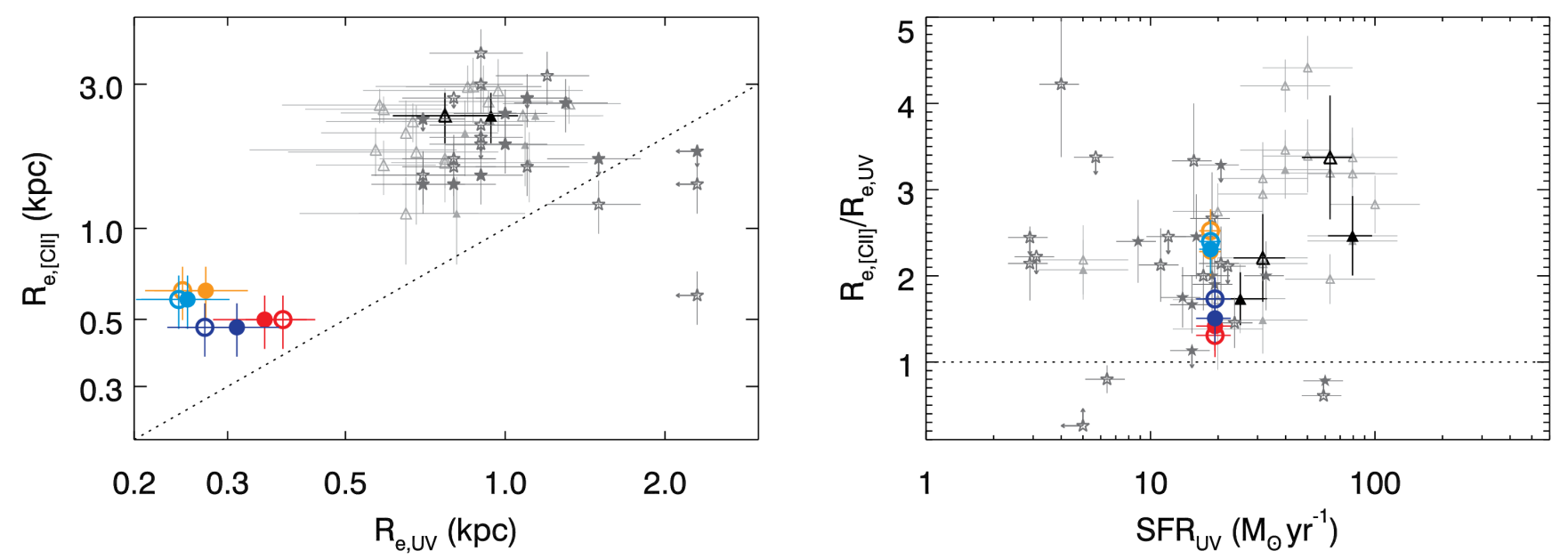

Figure 4. Physical sizes of mock and observed galaxies. Left: comparison of the [CII] and rest-frame UV circularized effective radii. Literature results from the ALPINE survey (Fujimoto et al. 2020) are reported in gray, with open and filled triangles indicating radii measured in the F814W and F160W bands respectively. The black empty and filled triangles indicate the median of the literature data (for the F814W and F160W bands) and their error bars represent the standard error of the median. Literature results from the collection of Carniani et al. (2018, including data from Capak et al. 2015, Barisic et al. 2017, Faisst et al. 2017, Willott et al. 2015, Jones et al. 2017, Ouchi et al. 2013, Matthee et al. 2017, Pentericci et al. 2016, Smit et al. 2018, Maiolino et al. 2015, Ota et al. 2014, Carniani et al. 2017, Inoue et al. 2016) are shown as gray stars. Empty stars indicate multi-component systems, whereas filled stars indicate individual galaxies. Our measurements for the clumpy galaxy (orange and cyan circles) and merger (red and blue circles) are reported. The orange and red circles indicate a spatial resolution of $\sim 0.15$ ", whereas the cyan and blue circles represent the lensed case (resolution $\sim 0.05 ")$. We report the radii measured on the rest-frame $\left(z^{\prime}\right)$ and optical $(H)$ bands (empty and filled circles), to compare with the literature. The equality $R_{e,[C I I]}=R_{e, U V}$ is shown (dotted black line). Right: [CII]-to-UV circularized effective radii ratio as a funcion of UV SFR. The black stars indicate the median ratios in bins of SFR. Colors and symbols are the same as in the left panel.

cal sizes measured in the $H$ band $^{9}$ (Fujimoto et al. 2020). In Figure 4 (left panel) we compare the sizes measured in the $z, H$, and [CII] maps of our galaxies with the results from Fujimoto et al. (2020). We find that the [CII] is systematically more extended than the rest-frame UV emitting regions, with [CII]-to-UV size ratios similar to those reported in the literature. We also find that the $\mathrm{R}_{\mathrm{e},[\mathrm{CII}]} / \mathrm{R}_{\mathrm{e}, \mathrm{z}}$ ratio is $\sim 20-30 \%$ smaller than the $R_{e,[C I]} / R_{e, H}$ ratio, in agreement with literature results.

Our galaxies however seem to be more compact than those observed by Fujimoto et al. (2020), having both UV and $[\mathrm{CII}]$ effective radii a factor $\sim 2.5$ smaller than those reported in the literature. This might be due to the fact that the two stages of Althæa that we have analyzed were originally found at higher redshift $(z \gtrsim 7$, see Section 3$)$, where galaxies are expected to have smaller sizes, at fixed stellar mass (Allen et al. 2017, Whitney et al. 2019). More simulations are needed to understand the origin of this potential discrepancy with observations.

We further investigated whether the ratio of the [CII]to-UV radii could be related to the galaxy SFR (Figure

\footnotetext{
9 The [CII] observations of the ALPINE survey have been performed with a spatial resolution of $\sim 0.7$ " (Le Fèvre et al. 2019). To understand whether the higher spatial resolution of our mock maps was biasing the comparison with the ALPINE results, we have created a set of [CII] maps with $\sim 0.7$ " angular resolution. The $[\mathrm{CII}]$ effective radius that we retrieved is $800 \pm 160 \mathrm{pc}$, slightly larger than the one measured at higher resolution, but consistent within the uncertainties. We therefore conclude that the high angular resolution of the mock observations does not bias our comparison.
}

4, right panel). Here we consider the unobscured SFR as derived from the UV luminosity, without correction for dust extinction. We complemented the literature sample of Fujimoto et al. (2020) with that of Carniani et al. (2018), and compared with our mock observations. A trend of $\mathrm{R}_{\mathrm{e},[\mathrm{CII}]} / \mathrm{R}_{\mathrm{UV}}$ with SFR is observed when considering the galaxies from Fujimoto et al. (2020) only, but it is washed out when adding the sample from Carniani et al. (2018). We notice that while Fujimoto et al. (2020) excludes disturbed systems from their sample, a large fraction of the sources reported by Carniani et al. (2018) can be considered as mergers (Section 6). This could be a reason why the two samples show discrepant results about the existence of a $\mathrm{R}_{\mathrm{e},[\mathrm{CII}]} / \mathrm{R}_{\mathrm{e}, \mathrm{UV}}$ trend with SFR. This seems to be consistent with our mock observations showing that the clumpy disk and merger, despite having similar SFRs show significantly different ratios $\left(\mathrm{R}_{\mathrm{e},[\mathrm{CII}]} / \mathrm{R}_{\mathrm{UV}} \sim 2.5\right.$ for the clumpy disk and $\sim 1.5$ for the merger). More data on both the observational and theoretical side are needed to clarify whether the UVto-[CII] ratio indeed scales with the galaxy SFR.

We highlight that Fujimoto et al. (2020) reports circularized effective radii $\left(\mathrm{R}_{\mathrm{e} \text {, circ }}=\mathrm{R}_{\mathrm{e}} \sqrt{q}\right.$, where $q$ is the axis ratio). To perform a consistent comparison, in Figure 4 we also report the circularized effective radii of our galaxies, whereas in all other figures we show $\mathrm{R}_{\mathrm{e}}$. Given that the axis ratio of our galaxies is $q \gtrsim 0.7$, the difference between $R_{\mathrm{e}}$ and $\mathrm{R}_{\mathrm{e} \text {, circ }}$ is $\lesssim 20 \%$. 


\subsection{Galaxy substructure}

Our simulations show that galaxies in the early Universe do not appear simply as smooth disks, but they rather have complex substructures such as star-forming clumps, merging satellites, proto-spiral arms, and rings around the nucleus (Figure 1,2). From our mock observations we can assess whether these substructures are detectable or are lost due to the limited spatial resolution and sensitivity. It is also possible to identify the optimal observational bands for substructure detection, and compare data taken at different wavelengths.

In the following we denote "clumps" as star-forming regions arising from disk gravitational instabilities, and "satellites" as small galaxies of external origin merging with Althaca's disk. Note that satellites are embedded in their dark matter halo, whereas clumps are not (Kohandel et al. 2019). Finally, clumps detected in our mock maps are typically in virial equilibrium (Leung et al. 2019), and are therefore stable, self-gravitating structures.

We investigated whether the extended [CII] could be emitted by unresolved, dust-obscured satellites or clumps by comparing the effective radii estimated from mock observations with different spatial resolution. The [CII] and UV sizes that we measured are comparable in the lowand high-resolution cases (Figure 3), despite the presence of clumps that can only be resolved when the spatial resolution is $\sim 0.05$ " or better (see Section 5.2.1). Furthermore, our clumpy disk shows two clumps aligned along the galaxy major axis (Figure A1). They are below the detection threshold both in [CII] and UV maps, and they are only detected (and therefore deblended) at near- and mid-infrared bands. Nevertheless, the effective radius of the disk measured in the $H S T$ optical and JWST infrared bands is comparable within the uncertainties. This suggests that the presence of unresolved satellites and/or clumps does not substantially bias the measurement of the disk effective radius. Given the fact that we estimated the $[\mathrm{CII}]$ size with the same procedure as the optical one, we can as well conclude that the [CII] extended emission is not biased by the presence of undetected substructures.

We conclude that at $z \sim 6$ the [CII] emission is intrinsically more extended than the optical one likely due to the joint effect of the carbon photoionization produced by UV photons emitted by the galaxy itself and penetrating in the surrounding neutral medium, and by the outflows produced by supernovae and massive stars that expel [CII] outside the disk.

\subsubsection{Spatial resolution}

We determined the number of clumps and satellites detected in the maps with the original resolution of the simulation ( 25 pc) by using Sextractor (Bertin \& Arnouts 1996, Section 4). Both our clumpy disk and merger show a similar number of substructures (Figure 5), between 7 and 11 depending on the observing band. When we consider the low- and high-resolution galaxy maps (resolution $\sim 0.15$ " and $\sim 0.05$ " respectively), the number of detected substructures drastically decreases. The clumps that are closer to the galaxy nucleus are blended and cannot be detected against the galaxy disk. The fainter and smaller clumps and satel- lites are instead undetected due to the lack of sensitivity and resolution of the mock observations. Only the substructures with high enough contrast against the galaxy disk or the background (e.g. those that are further away from the nucleus and/or brighter) can be detected.

We do not detect substructures in the low-resolution clumpy galaxy case, which appears as a smooth Sérsic disk (Figure A2). In the merger case we only detect one substructure in the near-infrared $J W S T /$ NIRCam band and in the [CII] map, but it does not appear at optical wavelengths. This is a merging satellite with stellar mass $\mathrm{M}_{\star}=1.3 \times 10^{9}$ $\mathrm{M}_{\odot}$, molecular gas mass $\mathrm{M}_{\mathrm{H} 2}=0.4 \times 10^{7} \mathrm{M}_{\odot}$, and $\mathrm{SFR}=10.8$ $\mathrm{M}_{\odot} \mathrm{yr}^{-1}$. Its projected galactocentric distance $(\sim 2.5 \mathrm{kpc})$, and high contrast allowed us to deblend it from the disk and detect it.

When considering the high-resolution case instead (resolution 0.05") more substructures appear. The clumpy disk shows one clump, detected in the near- and mid-infrared bands. The merger shows two substructures in the optical and [CII] maps, and five at near- and mid-infrared wavelengths. The stellar mass of these substructures ranges between $M_{\star} \simeq(0.3-2.5) \times 10^{8} M_{\odot}$, their total gas mass $M_{\text {gas }} \sim(1-4) \times 10^{7} M_{\odot}$, and their $\mathrm{SFR} \simeq 0.2-1.0 \mathrm{M}_{\odot} \mathrm{yr}^{-1}$. The most massive one and the most distant from the galaxy nucleus is a satellite, whereas the innermost ones are clumps. We note however that even in our high-resolution cases, only the clumps that are laying in the outskirts of the disk are detectable, whereas the innermost ones have a too low contrast with the disk to be deblended and studied. We note however that the detection of clumps might be easier when targeting galaxies with larger effective radii than Althæa, as the contrast of the substructures with the galaxy disk is higher.

\subsubsection{Observing bands}

Finally, we investigate how the band used to carry out the observations affects the detection of galaxies substructure by comparing the number of clumps and satellites found in maps at different wavelengths. It seems that the nearand mid-infrared JWST maps are the most suitable ones for this study, as they show the highest number of detected substructures (Figure 5).

This can be seen when considering the SED of the individual substructures found in our mock maps. In Figure 6 we show that indeed those with the highest dust extinction (e.g. number 1 and 2 in the clumpy high-resolution case; number 3 and 4 in the merger high-resolution case) are undetected in the HST bands (Table 3). At optical wavelengths we could only detect the unobscured substructures (e.g. number 1 and 2 in the merger, high-resolution case) which are also metalpoor and/or gas-poor and, due to these reasons, are undetected in [CII] (Table 4). The JWST bands instead allow us to simultaneously detect both unobscured (metal-poor) and dust-obscured (metal-rich) substructures, being therefore ideal for the study of galaxy clumps and satellites.

The number of substructures detected at optical and sub-millimeter wavelengths is similar, although those found in the HST and ALMA [CII] maps are not co-spatial (see e.g. Figure A3). The morphology of the clumpy disk and merger appearing at optical and sub-millimeter wavelengths is the following: 

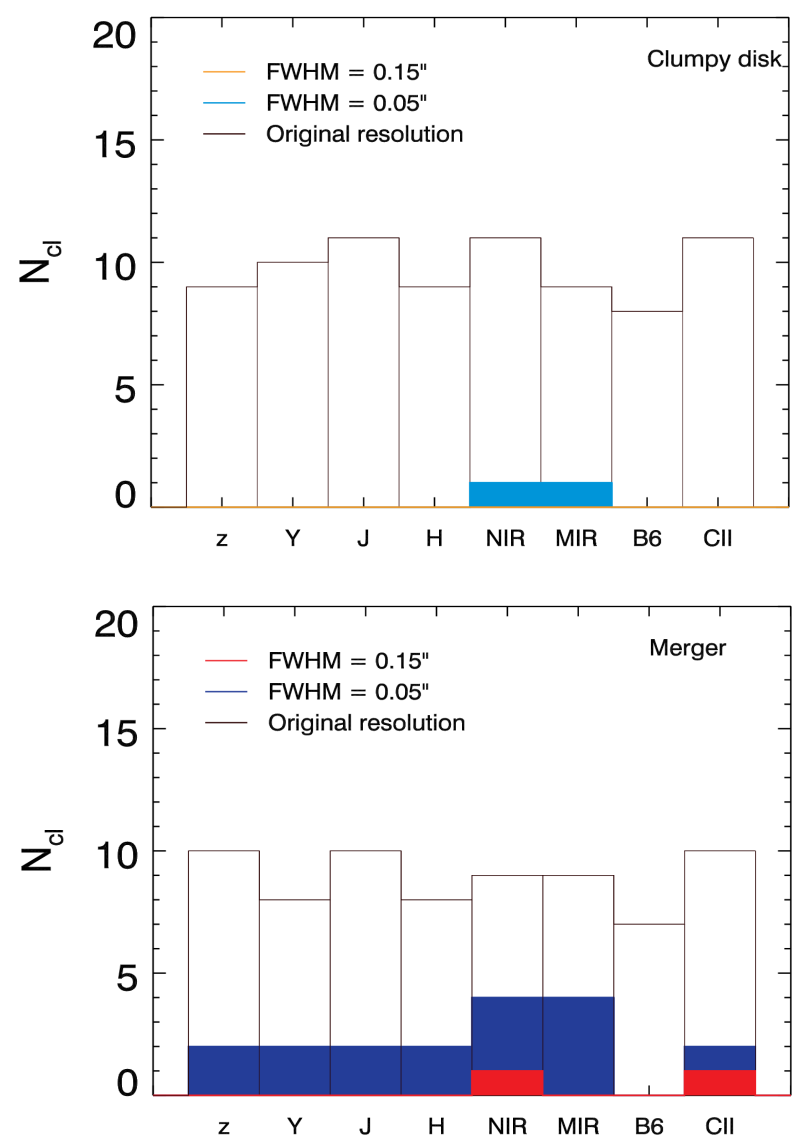

Figure 5. Number of substructures found in different observing bands and with different spatial resolution. Top: clumpy galaxy case. Bottom: merger case. The number of substructures found in the simulations with original resolution (black empty histogram), mock observations with $\sim 0.15$ " (red filled histogram), and $\sim 0.05 "$ (cyan and blue filled histogram) resolution are reported.

- Clumpy disk. The galaxy disk is detected in both HST optical and ALMA [CII] maps and the emissions are co-spatial. We do not detected substructures at these wavelengths (the only substructures are detected in the infrared $J W S T$ bands), irrespective of the spatial resolution (Figure $\mathrm{A} 1$ and A2).

- Merger. Also in this case, the galaxy disk is detected both in optical and [CII] bands, although a more complex morphology is observed. In the low-resolution case, only the galaxy disk is visible at optical wavelengths, whereas the [CII] map also shows an additional component. It is a small satellite (stellar mass $M_{\star}=1.3 \times 10^{9} M_{\odot}$, total gas mass $M_{\text {gas }}=3.4 \times 10^{9} M_{\odot}$ ) merging with the main galaxy (Figure A4). It has a distance of $\sim 2.5 \mathrm{kpc}$ and it is unresolved at the resolution of these observations ( 0.15 ", corresponding to $\sim 0.8 \mathrm{kpc}$ at this redshift).

More substructures are seen at $\sim 0.05$ " resolution (Figure A3). In particular, two clumps are detected at optical wavelengths (but not in [CII]) and, viceversa, other two structures are detected in [CII] (but not in the optical bands). The two optical substructures have a distance from the galaxy center of $0.7-0.8 \mathrm{kpc}$, whereas the two sub-mm substructures are found respectively at a distance of $\sim 0.8 \mathrm{kpc}$ and $\sim 2.5$ $\mathrm{kpc}$ from the galaxy center. The substructure found at 2.5 $\mathrm{kpc}$ from the galaxy disk is a satellite (the same detected also with spatial resolution $\sim 0.15$ "), whereas the others are clumps.

The origin of the optical versus [CII] displacement in our mocks is the role played by dust extinction and metallicity, as already pointed out by Vallini et al. (2015), Pallottini et al. (2017b), and Katz et al. (2017). The most dust-obscured structures are detected in [CII] but not in the optical bands and, viceversa, the most metal-poor ones are detected at rest-frame UV wavelengths but not in the submm (Figure 6).

\section{DISCUSSION}

In recent years increasingly large samples of high-redshift galaxies observed at both optical and sub-millimeter wavelengths with relatively high spatial resolution ( 0.15" - 0.5") have been reported (e.g. Mallery et al. 2012, Carilli \& Walter 2013, Willott et al. 2015, Maiolino et al. 2015, Capak et al. 2015, Inoue et al. 2016, Pentericci et al. 2016, Knudsen et al. 2016, Carniani et al. 2017, Barisic et al. 2017, Bradač et al. 2017, Carniani et al. 2018, Fujimoto et al. 2020). Some of them appear as isolated disks, whereas others show morphologies with multiple components. These studies have also shown that multiwavelength datasets are crucial to study these primordial systems, as various tracers (e.g. optical versus [CII]) reveal different morphologies, with some substructures being detected only at certain wavelength. As a result, some of these multi-component systems have co-spatial optical and sub-millimeter emission, whereas others show spatial offsets. In order to understand what is the nature of the multi-component systems currently observed in the literature (i.e. accreting satellites vs star-forming clumps), we compare their properties (separation of the multiple components, their spatial extent, their luminosity at different wavelengths) with those of the star-forming clumps and accreting satellite detected in the stages of Althæa analyzed in Sections 4 and 5 .

\section{Separation of multiple components}

We compared the structure of our clumpy disk and merger with the morphology reported in the literature for galaxies observed with HST in the optical and ALMA in the submillimeter.

- Single-component systems. The $z=6.17$ galaxy (CLM1) observed by Willott et al. (2015) appears as an individual source with spatially coincident [CII] and optical emission. Other two similar cases have been observed by Smith et al. (2017) at $z=6.85$ and $z=6.81$, although the beam size of the ALMA observations was 1.1 " $\times 0.7$ ", and data with better angular resolution would be needed to confirm these results. In the sample collected by Carniani et al. (2018), 60\% of the sources have a single-component morphology and they all show spatially-coincident optical and [CII] emission. Finally, both Knudsen et al. (2016) and 

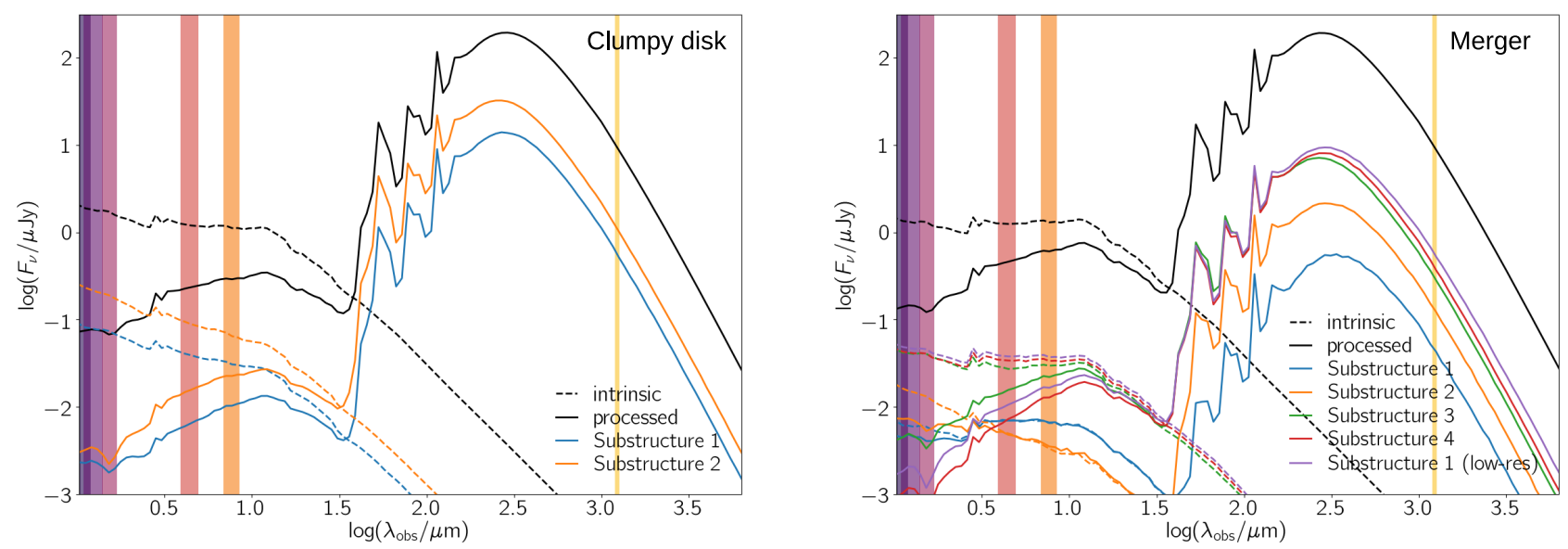

Figure 6. Spectral energy distribution of the clumpy galaxy and merger. We show the SED of the integrated galaxy (black curve) and those of the individual substructures found in our mock maps (colored curves). We display the intrinsic emission (dashed curves) and the "observed" one that also includes the effects of dust extinction (solid curves). The vertical colored strips indicate the observing bands used to create our mock maps $(H S T / z$ ', J, Y,H, JWST/NEAR-IR, MID-IR, ALMA/Band 6). Left panel: clumpy disk. Right panel: merger.

Table 4. Physical parameters of the detected substructures.

\begin{tabular}{|c|c|c|c|c|c|c|c|c|}
\hline Galaxy & Resolution & Component & $\begin{array}{c}M_{\star} \\
10^{8} \mathrm{M}_{\odot} \\
(4)\end{array}$ & $\begin{array}{c}\mathrm{SFR} \\
\mathrm{M}_{\odot} / \mathrm{yr} \\
(5)\end{array}$ & $\begin{array}{c}M_{\mathrm{g}} \\
10^{8} \mathrm{M}_{\odot} \\
(6)\end{array}$ & $\begin{array}{c}M_{\mathrm{H} 2} \\
10^{6} \mathrm{M}_{\odot} \\
(7)\end{array}$ & $\begin{array}{c}Z \\
Z_{\odot} \\
(8)\end{array}$ & $\begin{array}{l}A_{V} \\
(9)\end{array}$ \\
\hline \multirow[t]{2}{*}{ Clumpy } & High-resolution & Substructure 1 & 1.3 & 4.2 & 0.2 & 1.1 & 0.2 & 2.2 \\
\hline & & Substructure 2 & 2.7 & 3.9 & 0.9 & 0.8 & 0.02 & 2.1 \\
\hline \multirow[t]{5}{*}{ Merger } & Low-resolution & Substructure 1 & 4.7 & 1.7 & 1.6 & 1.9 & 0.1 & 1.5 \\
\hline & High-resolution & Substructure 1 & 0.4 & 0.2 & 0.1 & 0 & 0.1 & 0.1 \\
\hline & & Substructure 2 & 0.3 & 0.2 & 0.4 & 0.5 & 0.02 & 0.1 \\
\hline & & Substructure 3 & 1.6 & 0.3 & 0.3 & 0 & 0.1 & 0.8 \\
\hline & & Substructure 4 & 2.5 & 1.0 & 0.3 & 0.5 & 0.2 & 1.9 \\
\hline
\end{tabular}

Columns: (1) Galaxy: clumpy disk or merger. (2) Resolution: low- ( 0.15") or high-resolution ( 0.05"). (3) Component: substructure found in the mock maps. (4) Stellar mass. (5) Star formation rate. (6) Gas mass. (7) Molecular gas mass. (8) Metallicity. (9) Dust extinction.

Bradač et al. (2017) report the discovery of lensed galaxies (magnification $\mu=11.4 \pm 1.9$ and $\mu=5.0 \pm 0.3$ respectively) showing spatially coincident $[\mathrm{CII}]$ and $Y$ band emission. No substructures are found in these galaxies, despite lensing allows to reach higher spatial resolutions.

- Multi-component systems. Among the $z=5-7$ sources presented by Carniani et al. (2018) observed both with HST and ALMA, 40\% show multi-component morphologies. The substructures are separated by $\gtrsim 2$ kpc. For half of these targets the optical and sub-millimeter emissions are not co-spatial, with some components visible at optical wavelengths, whereas others detected in [CII].

The literature galaxies showing a single component are comparable to our clumpy disk case. The comparison with our simulations suggests that they are likely isolated, undisturbed disk galaxies, with a rather homogeneous distribution of dust and metals, allowing the detection of both optical and [CII] emission. Only the galaxy disk is detected in these cases and no substructures are observed.
The comparison with our mock observations suggests instead that the multi-component systems are likely galaxies undergoing mergers, rather than star-forming clumps formed in-situ in the galaxy disk. The substructures reported in the literature in fact have offsets $\gtrsim 2 \mathrm{kpc}$ (Carniani et al. 2018), consistent with the distance of the satellite from the galaxy disk $(\sim 2.5 \mathrm{kpc})$ in our merger case. Some of the satellites might be detected only in the optical or [CII], giving rise to the observed spatial offsets, due to different metallicity or dust content, as it is the case for Althæa (Section 5.2.2).

We point out that the galaxy disk in our mock observations is always detected both in the optical and submillimeter bands, so we do not have extreme cases of completely displaced optical and [CII] emission as the one reported by, e.g. Maiolino et al. (2015); Carniani et al. (2017). While strong differences in the dust and metallicity content of the subcomponents in this system could explain the observed offsets, strong feedback cleaning the most vigorous star-forming regions might also play a role in this case, 
as suggested by Maiolino et al. (2015) and Gallerani et al. (2018). More simulations of high-redshift galaxies are required to assemble a statistically significant sample, investigate whether simulated systems with completely offset optical and sub-millimeter emissions exist, and understand what is the role played by feedback in the assembly of early galaxies.

\section{Spatial extent}

Current observations reporting multiple substructures have been performed at $z \sim 5-7$ with angular resolution $\gtrsim 0.15$ ". Most of these substructures are spatially resolved and have rest-frame UV sizes $R_{e, U V} \gtrsim 0.7 \mathrm{kpc}$ and infrared sizes $\mathrm{R}_{\mathrm{e},[\mathrm{CII}]} \gtrsim 1 \mathrm{kpc}$ (Carniani et al. 2018). Their sizes are comparable to those measured for individual, isolated galaxies (Figure 4). This suggests that the multi-component systems currently reported in the literature are likely merging galaxies rather than disks hosting star-forming clumps formed in-situ. Additionally, in Section 5.2.1 we have shown that spatial resolution is key to study galaxies' substructure. Althæa's star-forming clumps are detected only in the highresolution maps (resolution $\sim 0.05$ "), whereas in the lowresolution case only the galaxy disk and a satellite are visible. This indicates that higher resolution than currently achieved in observations is needed to detect the internal structure of galaxies.

\section{UV and [CII] luminosity}

Finally, we compared the optical and [CII] luminosity of single- and multi-component systems from the literature, with the disk and substructures identified in the mock maps of our clumpy disk and merger. In local galaxies a tight relation between the $[\mathrm{CII}]$ and rest-frame UV luminosity (or equivalently the unobscured star formation rate, $S_{F R}$ ) has been observed (e.g. Pineda et al. 2013, De Looze et al. 2014, Kapala et al. 2015, Herrera-Camus et al. 2015). Recently it has been investigated whether the same relation holds also at high redshift $(z>5)$.

Several studies have reported that high-redshift galaxies seem to be more scattered and often [CII]deficient with respect to local sources (Willott et al. 2015; Pentericci et al. 2016; Bradač et al. 2017; Carniani et al. 2017; Harikane et al. 2018, 2019; for a theoretical interpretation see Ferrara et al. (2019) and Pallottini et al. 2019). However most of these studies did not consider the multicomponent nature of high-redshift galaxies and reported them as single sources in the [CII] luminosity - SFR plane. Carniani et al. $(2018,2020)$ showed that when associating each [CII] subcomponent with its proper UV counterpart (when detected), the high-redshift sources follow on average the local $\mathrm{L}_{[\mathrm{CII}]}$ - SFR relation. They estimate the dispersion of the high-redshift relation to be 1.8 times larger than the one reported by De Looze et al. (2014) for local galaxies, but no systematic offsets are observed. As discussed by Carniani et al. (2018), the larger dispersion at high-redshift might be explained by the presence of multicomponent and complex systems in different evolutionary stages that are not common in the local Universe. Similar results have been recently found also by Matthee et al. (2019) and Schaerer et al. (2020).
We have determined the sub-components location in the $\mathrm{L}_{[\mathrm{CII}]}-\mathrm{L}_{\mathrm{UV}}$ (or equivalently $\mathrm{SFR}_{\mathrm{UV}}$ ) plane (Figure 7) and compared our findings with literature works also reporting the unobscured SFR (De Looze et al. 2014; Carniani et al. 2018; Schaerer et al. 2020). Our galaxy disks and subcomponents (i.e. satellites and clumps) seem to lay on a consistent relation with respect to that reported in the literature for $z \sim 5-7$ sources, with clumps and the satellite typically being 10 times fainter than the disk.

Figure 7 also shows that most of the substructures detected in our mock observations have UV and [CII] luminosities that are below the detection threshold of current observations. We therefore conclude that most of the multicomponent systems reported in the literature are galaxies undergoing major mergers, rather than small satellites or clumps that, as shown by our simulations, would have lower luminosity. This is also consistent with their relatively large sizes $(\gtrsim 0.15$ " $\sim 1 \mathrm{kpc})$ and typical separation $(\gtrsim 2.5 \mathrm{kpc})$.

\subsection{Insights on the mass assembly of early galaxies}

Detecting star-forming clumps embedded in the disk of early galaxies is key to determine the fraction of clumpy sources and the contribution of hierarchical merging and insitu secular growth to the mass assembly of early galaxies. Shibuya et al. (2016) analyzed a sample of Lyman Break Galaxies at $z=4-8$ with available multi-band HST photometry and found $\sim 15 \%-20 \%$ of their sample galaxies to show substructures at these early times. When complementing this dataset with observations of lower-redshift galaxies $(z \sim 0-3)$ they find that the fraction of multi-component systems $\left(f_{\text {multi }}\right)$ reaches a peak at $z \sim 2\left(f_{\text {multi }} \sim 60 \%\right)$ and then declines again towards lower redshift $\left(f_{\text {multi }} \sim 40 \%\right.$ at $z \sim 1)$. They compared the redshift evolution of $f_{\text {multi }}$ with the expected evolution of minor and major merger fractions (Lotz et al. 2011). They found that mergers cannot fully explain the observed trend of $f_{\text {multi }}$ and that violent disk instability giving rise to in-situ massive clumps seems to play a major role in galaxy mass assembly. The fact that the $f_{\text {multi }}$ evolution with redshift seems to closely follow the star formation rate density evolution (Madau \& Dickinson 2014) reinforces this scenario.

However, the results by Shibuya et al. (2016) at $z \gtrsim 4$ are based on observations of a very specific galaxy population (i.e. Lyman Break Galaxies). Due to their sample selection and the lack of sub-millimeter data, some dust-obscured substructures might have not been detected and therefore the fraction of multi-component systems at these redshifts might be higher. In the sample by Carniani et al. (2018) that combines optical and [CII] observations, the fraction of multi-component systems is $\sim 40 \%$ at $z \sim 5-7$ and most of them seem to be consistent with being major (or possibly minor) mergers (Section 6).

In the near future it will be therefore key to acquire multi-wavelength observations of statistical samples of highredshift galaxies with exquisite spatial resolution to better constrain the evolution of $f_{\text {multi }}$ with redshift, understand what are the properties and nature of individual substructures (i.e. star-forming clumps vs merging satellites), and clarify what are the mechanisms driving galaxy formation at early epochs. 


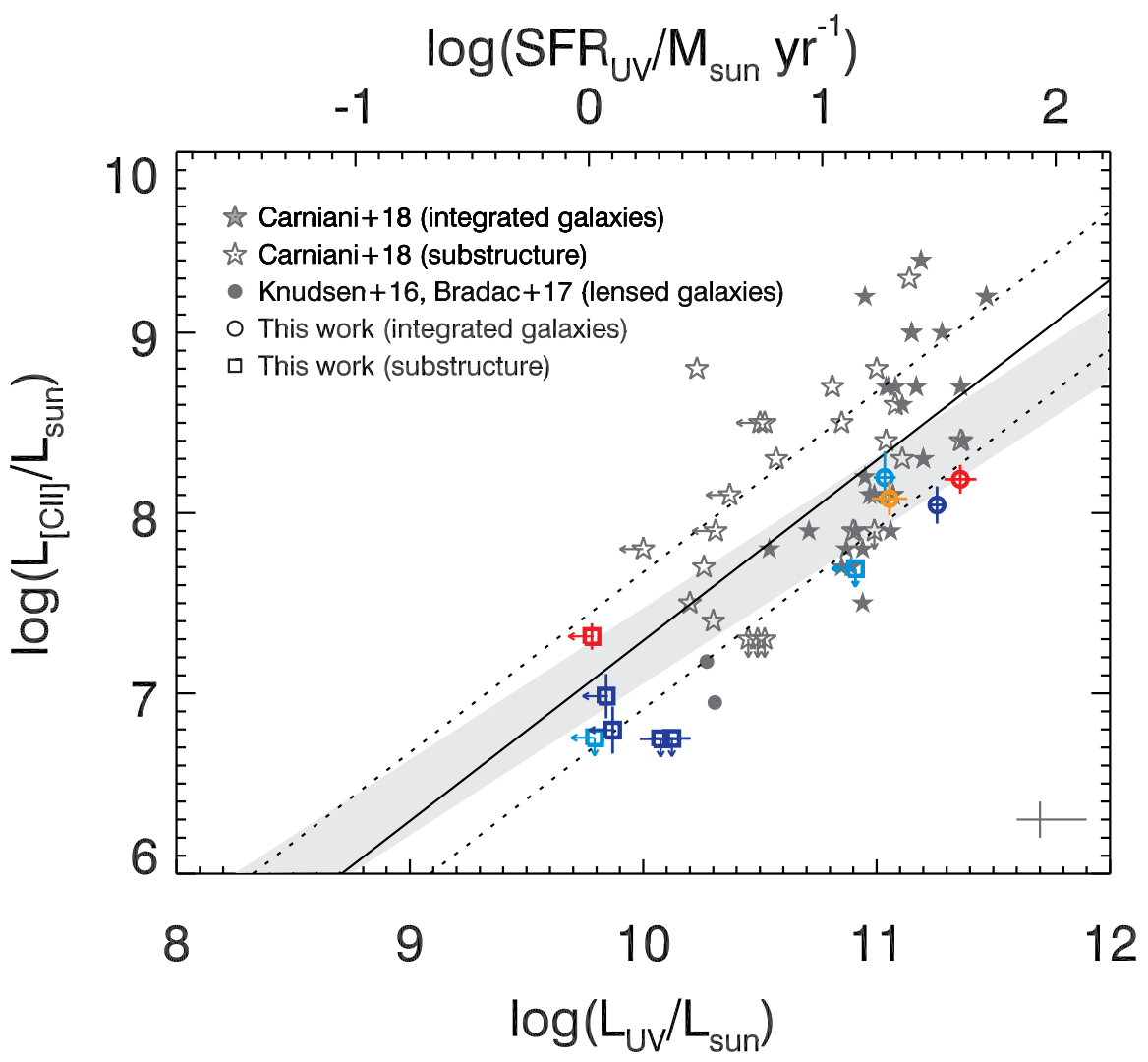

Figure 7. Comparison of the [CII] and UV luminosity of disks and substructures. Literature results for non-lensed targets (Carniani et al. 2018, and references therein) are reported as gray empty and filled stars to indicate respectively individual substructures and the total luminosity of the system (given by the sum of the luminosity of all related substructures). Literature results for lensed targets (Knudsen et al. 2016; Bradač et al. 2017) are reported as gray filled circles. Typical error bars are shown in the bottom right corner. Measurements and upper limits for our substructures (colored squares) and disks (colored circles) are shown (the colors are the same as in previous Figures). We report the relation between the [CII] luminosity and the unobscured SFR from De Looze et al. 2014 (black line) and its $1 \sigma$ standard deviation (dotted black lines). The gray filled area represents the relation between SFR (from optical SED fitting) and [CII] luminosity for the ALPINE galaxy sample (Schaerer et al. 2020).

With current facilities very high spatial resolution can be achieved by observing lensed sources (with magnification factor $\mu \gtrsim 10)$. However assembling large samples of lensed $z \sim 5-7$ galaxies is challenging. Furthermore, lensed sources are preferentially low mass, compact galaxies, that are expected to be less clumpy than more massive, larger targets (Bournaud et al. 2014; Shibuya et al. 2016; Guo et al. 2018). Likely for this reason no substructures have been detected in the lensed $z \sim 6$ galaxies observed so far with both HST and ALMA by Knudsen et al. (2016) and Bradač et al. (2017). To date only Vanzella et al. (2019) reports one lensed target at $z=6.143$ that shows internal substructure; the target is a highly magnified $(\mu \sim 20)$ dwarf galaxy hosting an extremely dense star-forming region (size $<13$ pc, stellar mass $<10^{6} \mathrm{M}_{\odot}$ ) ; this young (age $<10-100$ $\mathrm{Myr})$, moderately dust-obscured $(\mathrm{E}(\mathrm{B}-\mathrm{V})<0.15)$ star cluster was identified in a deep $H S T$ pointing and spectroscopically confirmed with adaptive optics-assisted MUSE observations. No submillimeter data showing the gas and dust content of this target are currently available, but they would be key to gain a complete picture of the internal structure of this galaxy.
With the next generation of telescopes (e.g. JWST, ELT) and instruments (e.g. MAVIS, the new adaptive optics-assisted visible imager and spectrograph proposed for the Very Large Telescope) it will be possible to investigate the internal structure of non-lensed targets (e.g. an angular resolution of $\sim 0.03$ " -0.05 " will be achieved at $\lambda \sim 0.7-1.5 \mu \mathrm{m}$ with $J W S T /$ NIRCam and $\sim 0.02 "$ in $V$ band with VLT/MAVIS), collecting statistical samples and understanding what is the role of secular evolution in the mass assembly and evolution of early galaxies. The future facilities operating at infrared wavelengths will also allow the community to detect dust-obscured clumps and satellites, finding the rest-frame optical counterpart of currently detected [CII] substructures (e.g. with the JWST/NIRCam near- and mid-infrared filters). This is seen in our mock observations as well, where the dust-obscured satellite lacking a rest-frame UV counterpart is instead detected in the JWST maps (Section 5.2). An additional piece of information will come from spatially-resolved metallicity measurements. By combining ALMA observations of the FIR [OIII] $52 \mu \mathrm{m}$ (or [OIII $] 88 \mu \mathrm{m})$ emission with JWST optical hydrogen lines (e.g. $\mathrm{H} \alpha, \mathrm{H} \beta, \mathrm{Pa} \alpha$ ) it will be possible to conduct spatially- 
resolved gas-phase metallicity measurements on sub-galactic scales (Jones et al. 2020). This will help distinguishing mergers from clumpy disks, get insights into gas mixing and feedback processes, and constrain the contribution of mergers and in-situ growth to the early assembly of galaxies.

\section{SUMMARY}

We have analyzed two stages of Althæa, a typical $z \simeq 6 \mathrm{Ly}$ man Break galaxy found in the SERRA zoom-in cosmological simulation suite. In the first snapshot Althæa appears as a clumpy disk, whereas in the second it is undergoing a merger with a small satellite (stellar mass ratio 1:8). We created mock optical $\left(z^{\prime}, Y, J\right.$, and $H H S T$-like), infrared (NIRCam/F444W and MIRI/F770W JWST-like) and submillimeter (Band 6 and [CII] ALMA-like) observations. We performed a $2 \mathrm{D}$ morphological analysis, considering maps with different angular resolutions ( 0.15 " and 0.05 "), and we deblended the emission of the galaxy disks from that of substructures (merging satellites or star-forming clumps). We found that:

- Our mock galaxies show $[\mathrm{CII}]$ effective radii $~ 1.5-$ 2.5 times larger than the optical ones. This is consistent with recent findings from the literature (e.g. Carniani et al. 2018, Fujimoto et al. 2020). We conclude that the observed [CII] halos arise from the joint effect of stellar outflows and carbon photoionization by the galaxy UV field, rather than from the emission of unresolved nearby satellites (Gallerani et al. 2018; Pizzati et al. 2020).

- With a spatial resolution of $\sim 0.15$ " we detect only one merging satellite at a distance of $\sim 2.5 \mathrm{kpc}$ from the galaxy nucleus. Star-forming clumps are instead embedded in the galaxy disk (distance $\lesssim 1 \mathrm{kpc}$ ). We show that better resolution $(\sim 0.05$ ") is required to detect these substructures at $z \sim 6$.

- Star-forming clumps found in our mock observations follow the local $\mathrm{L}_{[\mathrm{CII}]}-\mathrm{SFR}_{\mathrm{UV}}$ relation reported in the literature for galaxy disks, but sample the low-luminosity $\left(\mathrm{L}_{[\mathrm{CII}]} \lesssim 10^{7.5} \mathrm{~L} \odot_{\odot}\right)$, low-SFR $\left(\mathrm{SFR}_{\mathrm{UV}} \lesssim 3 \mathrm{M}_{\odot} \mathrm{yr}^{-1}\right)$ tail of the distribution.

- Only clumps with low dust extinction $\left(A_{V} \simeq 0.1\right)$ are detectable in the HST-like UV bands, whereas the dustobscured $\left(A_{V} \sim 1\right)$ and metal-rich ones are detected in [CII] maps. The JWST bands seem to be the most suitable ones to detect substructures thanks to their simultaneous sensitivity to both low-metallicity and dust-obscured regions that are bright at infrared wavelengths.

- By comparing the spatial extent, UV and [CII] luminosity, and separation of the substructures found in the multicomponents systems reported in the literature at $z \sim 5-7$ (Carniani et al. 2018), we conclude that current observations are likely detecting galaxies undergoing major mergers, rather than their internal star-forming clumps. Future telescopes (e.g. JWST, ELT) and instruments (e.g. VLT/MAVIS) with better sensitivity and spatial resolution will allow us to study star-forming clumps in $z \sim 6$ galaxies and quantify their contribution to the mass assembly of early galaxies.

\section{ACKNOWLEDGEMENTS}

We thank the referee, Nick Gnedin, for his comments that improved the clarity of the manuscript. AF (PI), MK, AP, SC acknowledge support from the ERC Advanced Grant INTERSTELLAR H2020/740120. Any dissemination of results must indicate that it reflects only the author's view and that the Commission is not responsible for any use that may be made of the information it contains. This research was partly supported by the Munich Institute for Astro- and Particle Physics (MIAPP) of the DFG cluster of excellence "Origin and Structure of the Universe". Partial support from the Carl Friedrich von SiemensForschungspreis der Alexander von Humboldt-Stiftung Research Award (AF) is kindly acknowledged. AZ acknowledges hospitality from Scuola Normale Superiore where part of this work has been developed. We acknowledge the use of the Python programming language (Van Rossum \& de Boer 1991), Astropy (Astropy Collaboration et al. 2013), Cython (Behnel et al. 2011), Jupyter (Kluyver et al. 2016), Matplotlib (Hunter 2007), NumPy (van der Walt et al. 2011), PYMSES (Labadens et al. 2012), PyNBOdy (Pontzen et al. 2013), and SciPy (Virtanen et al. 2019).

\section{DATA AVAILABILITY}

The data underlying this article were accessed from the computational resources available to the Cosmology Group at Scuola Normale Superiore, Pisa (IT). The derived data generated in this research will be shared on reasonable request to the corresponding author.

\section{REFERENCES}

Agertz O., Kravtsov A. V., 2015, ApJ, 804, 18

Allen R. J., et al., 2017, ApJ, 834, L11

Astropy Collaboration et al., 2013, A\&A, 558, A33

Baes M., Camps P., 2015, Astronomy and Computing, 12, 33

Bakx T. J. L. C., et al., 2020, MNRAS, 493, 4294

Barisic I., et al., 2017, ApJ, 845, 41

Behnel S., Bradshaw R., Citro C., Dalcin L., Seljebotn D., Smith K., 2011, Computing in Science Engineering, 13, 31

Behrens C., Pallottini A., Ferrara A., Gallerani S., Vallini L., 2018, MNRAS, 477, 552

Behrens C., Pallottini A., Ferrara A., Gallerani S., Vallini L., 2019, MNRAS, 486, 2197

Bertin E., Arnouts S., 1996, A\&AS, 117, 393

Bournaud F., 2016, Bulge Growth Through Disc Instabilities in High-Redshift Galaxies. Vol. 418, Springer (arXiv: 1503.07660), doi:10.1007/978-3-319-19378-6_13

Bournaud F., et al., 2008, A\&A, 486, 741

Bournaud F., et al., 2014, ApJ, 780, 57

Bovino S., Grassi T., Capelo P. R., Schleicher D. R. G., Banerjee R., 2016, A\&A, 590, A15

Bradač M., et al., 2017, ApJ, 836, L2

Brammer G. B., et al., 2012, ApJS, 200, 13

Bruzual G., Charlot S., 2003, MNRAS, 344, 1000

Camps P., Baes M., 2015, Astronomy and Computing, 9, 20

Capak P. L., et al., 2015, Nature, 522, 455

Capelo P. R., Bovino S., Lupi A., Schleicher D. R. G., Grassi T., 2018, MNRAS, 475, 3283

Carilli C. L., Walter F., 2013, ARA\&A, 51, 105

Carniani S., et al., 2017, A\&A, 605, A42 
Carniani S., et al., 2018, MNRAS, 478, 1170

Carniani S., et al., 2020, arXiv e-prints, p. arXiv:2006.09402

Ceverino D., Dekel A., Mandelker N., Bournaud F., Burkert A., Genzel R., Primack J., 2012, MNRAS, 420, 3490

Conselice C. J., 2014, ARA\&A, 52, 291

De Looze I., et al., 2014, A\&A, 568, A62

De Looze I., et al., 2020, MNRAS,

Decataldo D., Pallottini A., Ferrara A., Vallini L., Gallerani S., 2019, MNRAS, 487, 3377

Dekel A., Sari R., Ceverino D., 2009, ApJ, 703, 785

Faisst A. L., et al., 2016, ApJ, 822, 29

Faisst A. L., et al., 2017, ApJ, 847, 21

Ferland G. J., et al., 2017, Rev. Mex. Astron. Astrofis., 53, 385

Ferrara A., Vallini L., Pallottini A., Gallerani S., Carniani S., Kohandel M., Decataldo D., Behrens C., 2019, MNRAS, 489, 1

Fisher D. B., et al., 2017, MNRAS, 464, 491

Förster Schreiber N. M., Shapley A. E., Erb D. K., Genzel R., Steidel C. C., Bouché N., Cresci G., Davies R., 2011, ApJ, 731,65

Fujimoto S., et al., 2019, ApJ, 887, 107

Fujimoto S., et al., 2020, arXiv e-prints, p. arXiv:2003.00013

Gallerani S., Pallottini A., Feruglio C., Ferrara A., Maiolino R., Vallini L., Riechers D. A., Pavesi R., 2018, MNRAS, 473, 1909

Gelli et al., subm. 2020,-, 0, 0

Genel S., et al., 2012, ApJ, 745, 11

Genzel R., et al., 2008, ApJ, 687, 59

Genzel R., et al., 2011, ApJ, 733, 101

Genzel R., et al., 2014, ApJ, 785, 75

Ginolfi M., et al., 2020, A\&A, 633, A90

Girard M., Dessauges-Zavadsky M., Schaerer D., Richard J., Nakajima K., Cava A., 2018, A\&A, 619, A15

Grassi T., Bovino S., Schleicher D. R. G., Prieto J., Seifried D., Simoncini E., Gianturco F. A., 2014, MNRAS, 439, 2386

Grogin N. A., et al., 2011, ApJS, 197, 35

Guo Y., et al., 2015, ApJ, 800, 39

Guo Y., et al., 2018, ApJ, 853, 108

Hahn O., Abel T., 2011, MNRAS, 415, 2101

Harikane Y., et al., 2018, ApJ, 859, 84

Harikane Y., et al., 2019, ApJ, 883, 142

Heckman T. M., González-Delgado R., Leitherer C., Meurer G. R., Krolik J., Wilson A. S., Koratkar A., Kinney A., 1997, ApJ, 482, 114

Herrera-Camus R., et al., 2015, ApJ, 800, 1

Hopkins P. F., Kereš D., Murray N., Quataert E., Hernquist L., 2012, MNRAS, 427, 968

Hopkins P. F., et al., 2018, MNRAS, 480, 800

Hunter J. D., 2007, Computing in Science Engineering, 9, 90

Immeli A., Samland M., Westera P., Gerhard O., 2004, ApJ, 611,20

Inoue S., Dekel A., Mandelker N., Ceverino D., Bournaud F., Primack J., 2016, MNRAS, 456, 2052

Jiang L., et al., 2016, ApJ, 816, 16

Jones G. C., et al., 2017, ApJ, 850, 180

Jones T., Sanders R., Roberts-Borsani G., Ellis R. S., Laporte N., Treu T., Harikane Y., 2020, arXiv e-prints, p. arXiv:2006.02447

Kapala M. J., et al., 2015, ApJ, 798, 24

Katz H., Kimm T., Sijacki D., Haehnelt M. G., 2017, MNRAS, 468, 4831

Kennicutt Jr. R. C., 1998, ApJ, 498, 541

Kluyver T., et al., 2016, Jupyter Notebooks, a publishing format for reproducible computational workflows, https://eprints.soton.ac.uk/403913/

Knudsen K. K., Richard J., Kneib J.-P., Jauzac M., Clément B., Drouart G., Egami E., Lindroos L., 2016, MNRAS, 462, L6

Kohandel M., Pallottini A., Ferrara A., Zanella A., Behrens C., Carniani S., Gallerani S., Vallini L., 2019, MNRAS, 487, 3007

Kohandel et al., subm. 2020, -, 0, 0
Kroupa P., 2001, MNRAS, 322, 231

Labadens M., Chapon D., Pomaréde D., Teyssier R., 2012, in Ballester P., Egret D., Lorente N. P. F., eds, Astronomical Society of the Pacific Conference Series Vol. 461, Astronomical Data Analysis Software and Systems XXI. p. 837

Laporte N., et al., 2017, ApJ, 837, L21

Le Fèvre O., Béthermin M., Faisst A., Capak P., Cassata P., Silverman J. D., Schaerer D., Yan L., 2019, arXiv e-prints, p. arXiv:1910.09517

Leitherer C., et al., 1999, ApJS, 123, 3

Leung T. K. D., et al., 2019, ApJ, 871, 85

Liang L., et al., 2019, MNRAS, p. 2072

Lotz J. M., Jonsson P., Cox T. J., Croton D., Primack J. R., Somerville R. S., Stewart K., 2011, ApJ, 742, 103

Lupi A., Bovino S., Capelo P. R., Volonteri M., Silk J., 2018, MNRAS, 474, 2884

Lupi A., Pallottini A., Ferrara A., Bovino S., Carniani S., Vallini L., 2020, arXiv e-prints, p. arXiv:2004.06118

Madau P., Dickinson M., 2014, ARA\&A, 52, 415

Maio U., Petkova M., De Lucia G., Borgani S., 2016, MNRAS, 460, 3733

Maiolino R., Mannucci F., 2019, A\&ARv, 27, 3

Maiolino R., et al., 2015, MNRAS, 452, 54

Mallery R. P., et al., 2012, ApJ, 760, 128

Mandelker N., Dekel A., Ceverino D., DeGraf C., Guo Y., Primack J., 2015, arXiv:1512.08791, MNRAS, submitted

Mandelker N., Dekel A., Ceverino D., DeGraf C., Guo Y., Primack J., 2017, MNRAS, 464, 635

Maraston C., Nieves Colmenárez L., Bender R., Thomas D., 2009, A\&A, 493, 425

Matthee J., et al., 2017, ApJ, 851, 145

Matthee J., et al., 2019, ApJ, 881, 124

McMullin J. P., Waters B., Schiebel D., Young W., Golap K., 2007, CASA Architecture and Applications. p. 127

Moody C. E., Guo Y., Mandelker N., Ceverino D., Mozena M., Koo D. C., Dekel A., Primack J., 2014, MNRAS, 444, 1389

Oklopčić A., Hopkins P. F., Feldmann R., Kereš D., FaucherGiguère C.-A., Murray N., 2017, MNRAS, 465, 952

Olsen K., et al., 2018, Galaxies, 6, 100

Ota K., et al., 2014, ApJ, 792, 34

Ouchi M., et al., 2013, ApJ, 778, 102

Pallottini A., Ferrara A., Gallerani S., Salvadori S., D'Odorico V., 2014, MNRAS, 440, 2498

Pallottini A., Ferrara A., Gallerani S., Vallini L., Maiolino R., Salvadori S., 2017a, MNRAS, 465, 2540

Pallottini A., Ferrara A., Bovino S., Vallini L., Gallerani S., Maiolino R., Salvadori S., 2017b, MNRAS, 471, 4128

Pallottini A., et al., 2019, MNRAS, 487, 1689

Pavesi R., Riechers D. A., Faisst A. L., Stacey G. J., Capak P. L., 2019, ApJ, 882, 168

Peng Y.-j., et al., 2010, ApJ, 721, 193

Pentericci L., et al., 2016, ApJ, 829, L11

Pineda J. L., Langer W. D., Velusamy T., Goldsmith P. F., 2013, A\&A, 554, A103

Pizzati E., Ferrara A., Pallottini A., Gallerani S., Vallini L., Decataldo D., Fujimoto S., 2020, MNRAS, 495, 160

Pontzen A., Rovskar R., Stinson G. S., Woods R., Reed D. M., Coles J., Quinn T. R., 2013, pynbody: Astrophysics Simulation Analysis for Python

Puech M., Hammer F., Flores H., Neichel B., Yang Y., 2009, A\&A, 493, 899

Ribeiro B., et al., 2017, A\&A, 608, A16

Rosdahl J., et al., 2018, MNRAS, 479, 994

Schaerer D., et al., 2020, arXiv e-prints, p. arXiv:2002.00979

Schmidt M., 1959, ApJ, 129, 243

Shibuya T., Ouchi M., Harikane Y., 2015, ApJS, 219, 15

Shibuya T., Ouchi M., Kubo M., Harikane Y., 2016, ApJ, 821, 72

Smit R., et al., 2018, Nature, 553, 178 
Smith J. D. T., et al., 2017, ApJ, 834, 5

Steidel C. C., Erb D. K., Shapley A. E., Pettini M., Reddy N., Bogosavljević M., Rudie G. C., Rakic O., 2010, ApJ, 717, 289

Tamburello V., Mayer L., Shen S., Wadsley J., 2015, MNRAS, 453,2490

Tamura Y., et al., 2019, ApJ, 874, 27

Teyssier R., 2002, A\&A, 385, 337

Tornatore L., Borgani S., Dolag K., Matteucci F., 2007, MNRAS, 382, 1050

Trebitsch M., Blaizot J., Rosdahl J., Devriendt J., Slyz A., 2017, MNRAS, 470, 224

Vallini L., Gallerani S., Ferrara A., Pallottini A., Yue B., 2015, ApJ, 813, 36

Vallini L., Ferrara A., Pallottini A., Gallerani S., 2017, MNRAS, 467, 1300

Vallini L., Pallottini A., Ferrara A., Gallerani S., Sobacchi E., Behrens C., 2018, MNRAS, 473, 271

Van Rossum G., de Boer J., 1991, CWI Quarterly, 4, 283

Vanzella E., et al., 2019, MNRAS, 483, 3618

Vika M., Bamford S. P., Häußler B., Rojas A. L., Borch A., Nichol R. C., 2013, MNRAS, 435, 623

Virtanen P., et al., 2019, arXiv e-prints, p. arXiv:1907.10121

Weingartner J. C., Draine B. T., 2001, ApJ, 563, 842

Whitney A., Conselice C. J., Bhatawdekar R., Duncan K., 2019, ApJ, 887, 113

Willott C. J., Carilli C. L., Wagg J., Wang R., 2015, ApJ, 807, 180

Wiseman P., Schady P., Bolmer J., Krühler T., Yates R. M., Greiner J., Fynbo J. P. U., 2017, A\&A, 599, A24

Wisnioski E., et al., 2011, MNRAS, 417, 2601

Wuyts E., Rigby J. R., Gladders M. D., Sharon K., 2014, ApJ, 781,61

Zanella A., et al., 2015, Nature, 521, 54

Zanella A., et al., 2019, MNRAS, 489, 2792

van der Walt S., Colbert S. C., Varoquaux G., 2011, Computing in Science Engineering, 13, 22
APPENDIX A: MORPHOLOGICAL STRUCTURE IN THE OPTICAL AND SUBMILLIMETER BANDS 


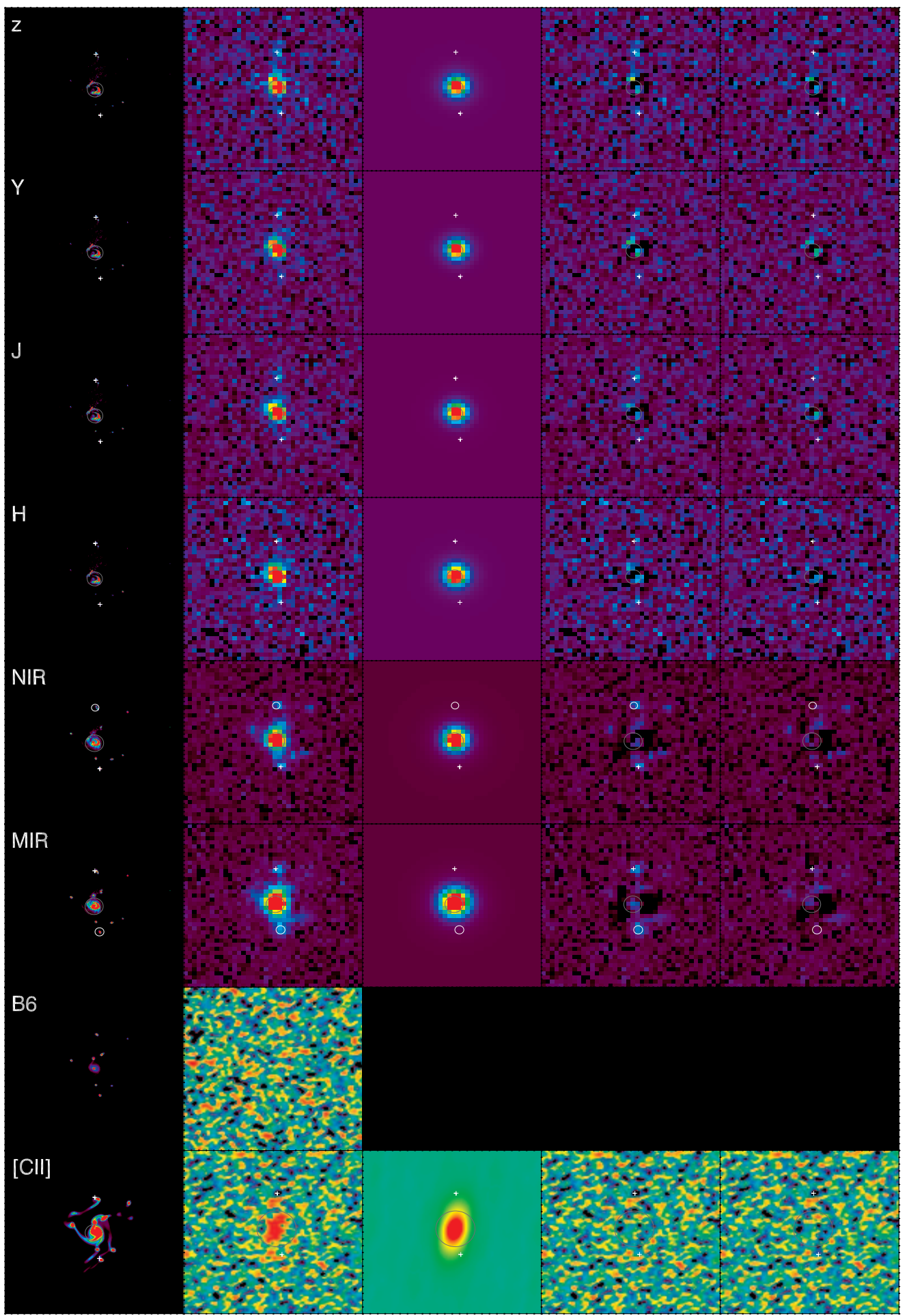

Figure A1. Mock observations for the disk galaxy with resolution 0.05". From top to bottom: HST/ACS F850LP ( $\left.z^{\prime}\right)$, HST/WFC3 F105W ( $Y$, F125W $(J)$, F160W $(H)$; JWST/NIRCam F444W $(N I R)$, JWST/MIRI F770W $(M I R)$; ALMA Band 6 continuum and the [CII] pseudo-narrow band emission line map. From left to right: map with the original simulation resolution, mock maps with the resolution of observations $(\sim 0.15 ")$, GALFITM model for the diffuse component (single Sérsic profile), residuals obtained subtracting the model (column 3) from the mock image (column 2), residuals obtained subtracting GALFITM best-fit model (including the diffuse Sérsic profile plus additional PSFs at the location of the substructures detected in the residuals shown in column 4) from the mock image (column 2). The dark gray circle indicates the center of the diffuse Sérsic profile and its radius is equivalent to the disk effective radius. The white crosses and circles indicate the center of the substructures (respectively detected with $\mathrm{S} / \mathrm{N}>3$ or non-detected). The radius of the white circles is equivalent to the FWHM of the PSF (if they are unresolved) or to the effective radius of the best-fit Sérsic profile (if they are resolved). Each stamp has a size of $0.6 " \times 0.6 "(\sim 3.4 \times 3.4 \mathrm{kpc}$ at $z \sim 6)$, we adopt the same color bar in columns 2 to 4 and an inverse hyperbolic sine scaling. 


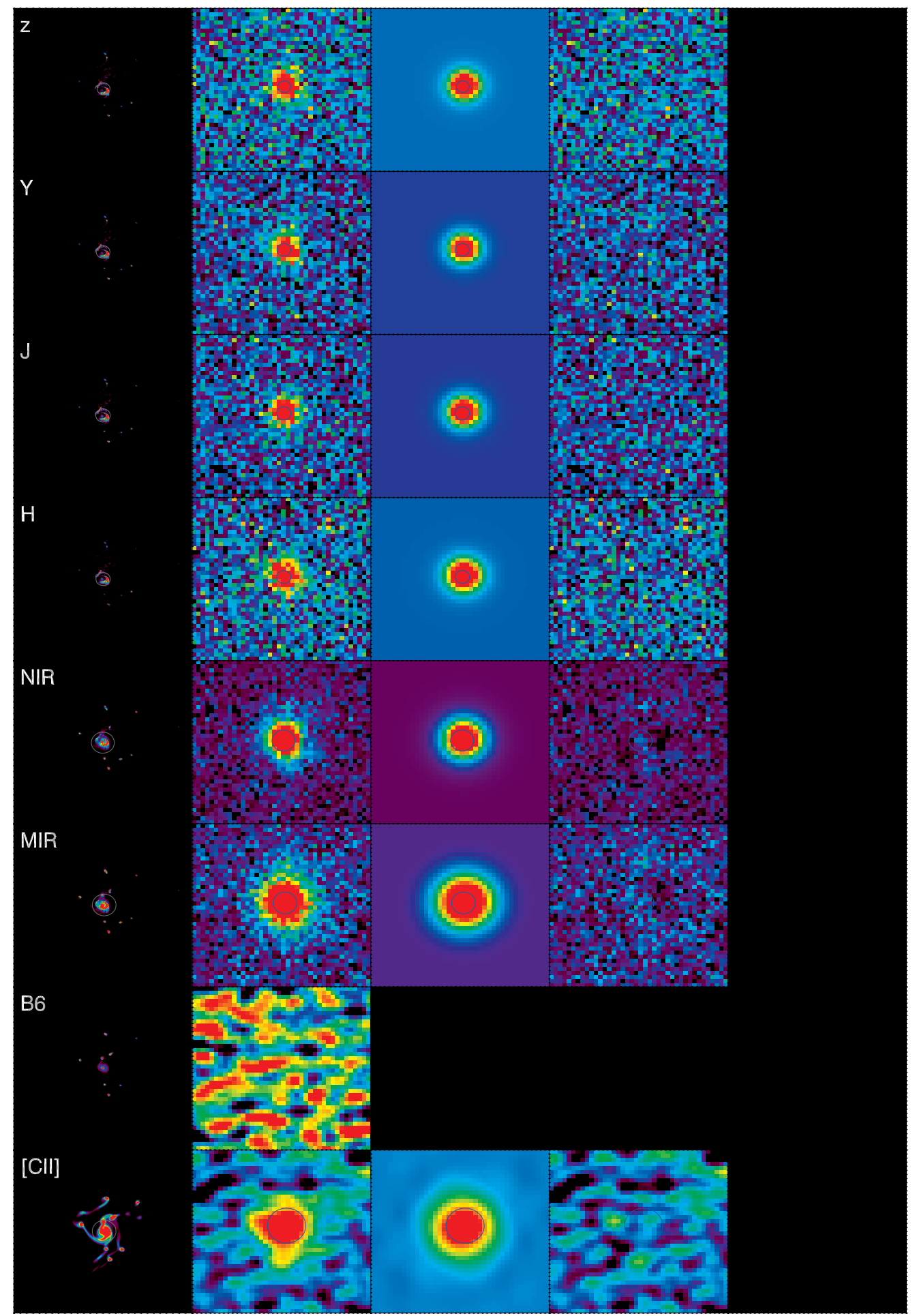

Figure A2. Mock observations for the disk galaxy with resolution 0.05". Images and symbols are the same as in Figure A1 


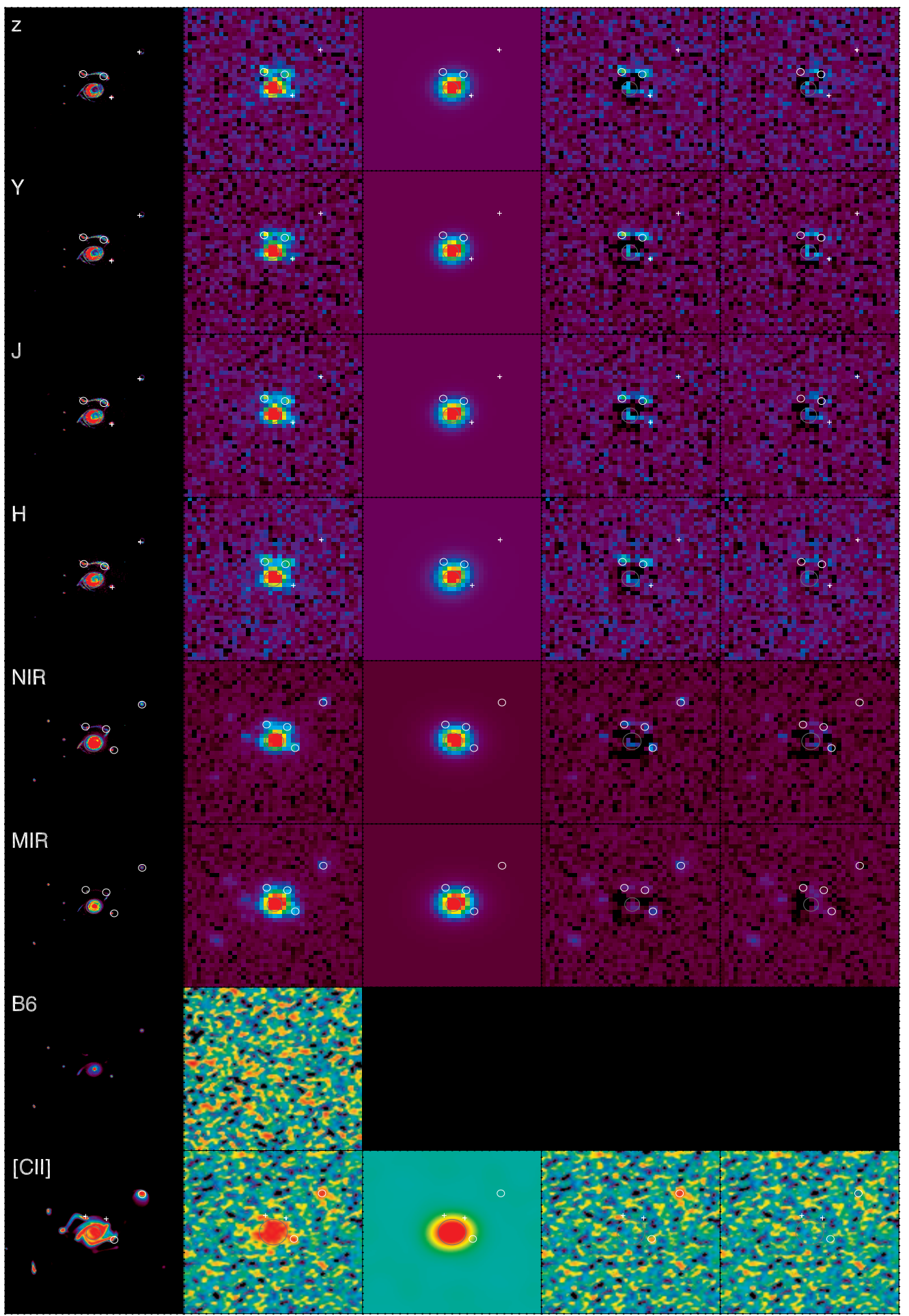

Figure A3. Mock observations for the merger with resolution 0.05". Images and symbols are the same as in Figure A1 


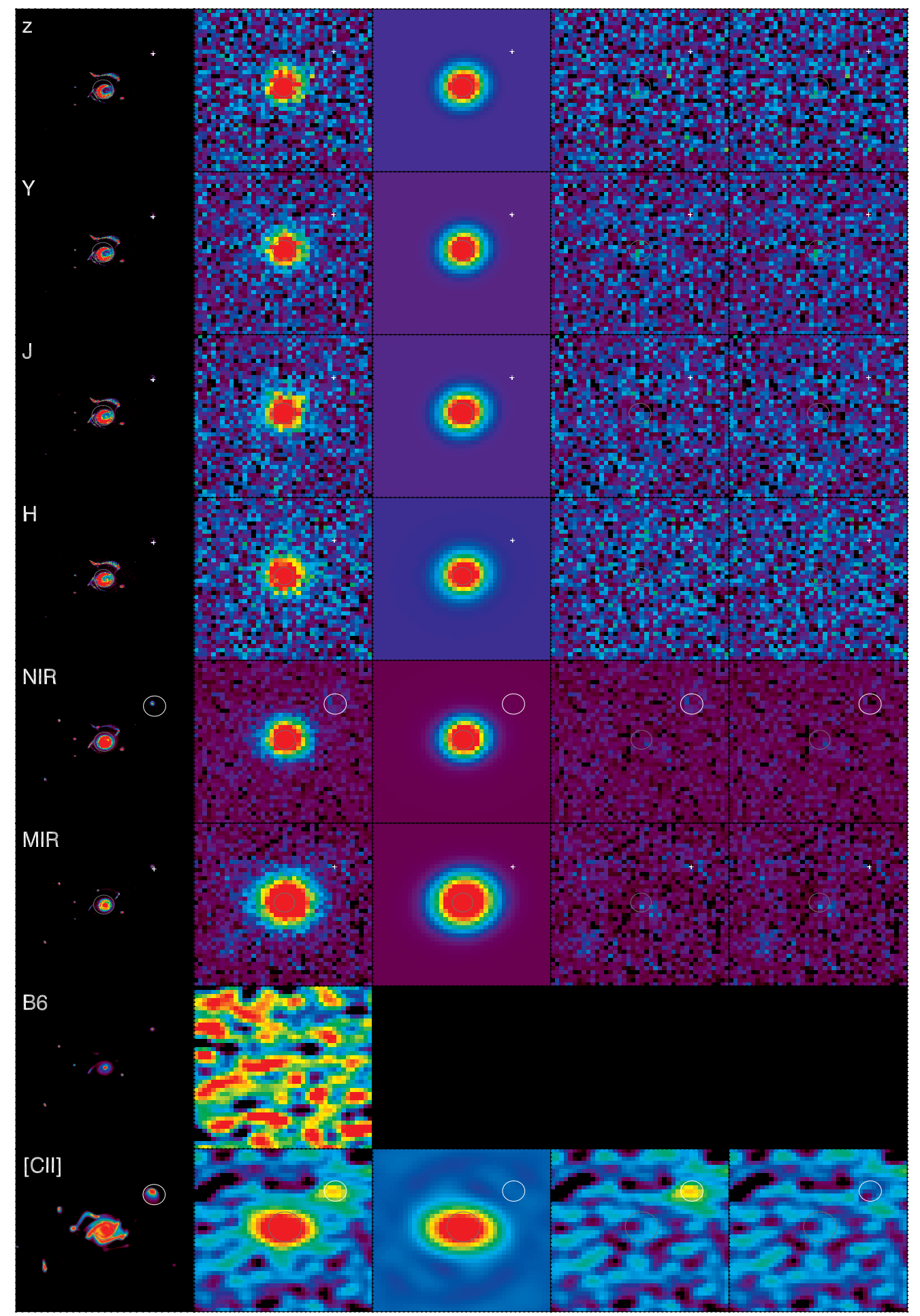

Figure A4. Mock observations for the merger with resolution 0.15". Images and symbols are the same as in Figure A1 
22 A. Zanella et al.

APPENDIX B: ESTIMATES OF THE

EFFECTIVE RADII

This paper has been typeset from a $\mathrm{T}_{\mathrm{E}} \mathrm{X} / \mathrm{LAT}_{\mathrm{E}} \mathrm{X}$ file prepared by the author. 

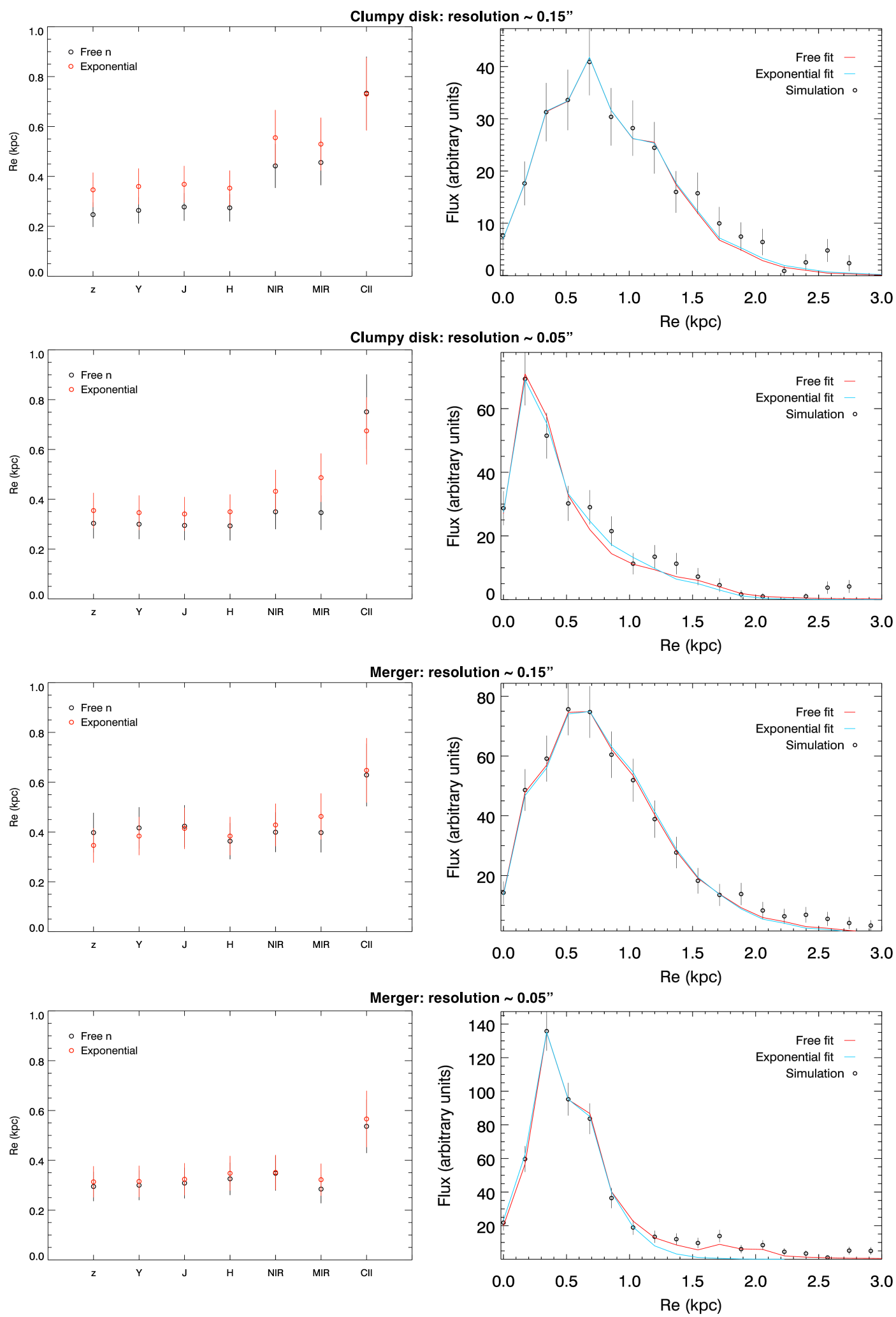

Figure B1. Comparison of the effective radius estimated when fitting the simulations with a Sérsic profile (with free Sérsic index) and with an exponential profile. Left column: effective radius measured in different bands. Each plot (from top to bottom) refers to mock observations of the clumpy galaxy and the merger, with different angular resolution (0.15" and 0.05"). The black (red) open circles indicate the effective radius obtained with a free Sérsic (exponential) profile fit. Right column: we show the one-dimensional surface brightness profile of the galaxies (black open circles with Poissonian error bars), as observed in the MIR band. We also show the free Sérsic (red curve) and exponential (cyan curve) profile fits. The effective radii measured with a free Sérsic and an exponential profile are consistent within the uncertainties and there are no systematic trends with the observing band. 